\title{
Noncanonical SQSTM1/p62-Nrf2 pathway activation mediates proteasome inhibitor resistance in multiple myeloma cells via redox, metabolic and translational reprogramming
}

\author{
Irene Riz ${ }^{1}$, Teresa S. Hawley ${ }^{2,3}$, Jeffrey W. Marsal ${ }^{1}$ and Robert G. Hawley ${ }^{1}$ \\ ${ }^{1}$ Department of Anatomy and Regenerative Biology, George Washington University, Washington, DC, USA \\ ${ }^{2}$ Flow Cytometry Core Facility, George Washington University, Washington, DC, USA \\ ${ }^{3}$ Flow Cytometry Core, National Cancer Institute, National Institutes of Health, Bethesda, MD, USA \\ Correspondence to: Robert G. Hawley, email: rghawley@gwu.edu \\ Keywords: multiple myeloma, carfilzomib, SQSTM1/p62, NFE2L2/Nrf2, translation initiation \\ Received: June 17,2016 Accepted: September 03, $2016 \quad$ Published: September 10, 2016
}

\section{ABSTRACT}

Multiple Myeloma (MM) is a B-cell malignancy characterized by the accumulation of clonal plasma cells in the bone marrow, with drug resistance being a major cause of therapeutic failure. We established a carfilzomib-resistant derivative of the LP-1 MM cell line (LP-1/Cfz) and found that the transcription factor NF-E2 p45-related factor 2 (Nrf2; gene symbol NFE2L2) contributes to carfilzomib resistance. The mechanism of Nrf2 activation involved enhanced translation of $\mathrm{Nrf} 2$ as well as its positive regulator, the autophagy receptor sequestosome 1 (SQSTM1)/p62. The eukaryotic translation initiation factor gene EIF4E3 was among the Nrf2 target genes upregulated in LP-1/ Cfz cells, suggesting existence of a positive feedback loop. In line with this, we found that siRNA knockdown of eIF4E3 decreased Nrf2 protein levels. On the other hand, elevated SQSTM1/p62 levels were due at least in part to activation of the PERKeIF2a pathway. LP-1/Cfz cells had decreased levels of reactive oxygen species as well as elevated levels of fatty acid oxidation and prosurvival autophagy. Genetic and pharmacologic inhibition of the Nrf2-EIF4E3 axis or the PERK-eIF2a pathway, disruption of redox homeostasis or inhibition of fatty acid oxidation or autophagy conferred sensitivity to carfilzomib. Our findings were supported by clinical data where increased EIF4E3 expression was predictive of Nrf2 target gene upregulation in a subgroup of patients with chemoresistant minimal residual disease and relapsed/ refractory MM. Thus, our data offer a preclinical rationale for including inhibitors of the SQSTM1/p62-Nrf2 pathway to the treatment regimens for certain advanced stage MM patients.

\section{INTRODUCTION}

Multiple myeloma (MM) is a B cell malignancy characterized by the clonal expansion of neoplastic plasma cells in the bone marrow. The introduction of novel agents such as proteasome inhibitors and immunomodulatory drugs has prolonged the lives of patients with $\mathrm{MM}$ over the past decade [1]. The first-in-class proteasome inhibitor bortezomib provided proof-of-principle that proteasome inhibition is an important therapeutic target in MM, leading to its FDA approval as frontline therapy in June 2008 [2]. Despite overall response rates of $100 \%$ in the setting of newly diagnosed disease [3], most MM patients ultimately relapse because the MM cells develop resistance to the treatment $[4,5]$. Carfilzomib, a second-generation proteasome inhibitor that exhibits enhanced selectivity for the proteasome [6], received full approval from the FDA for the treatment of patients with relapsed/refractory MM in January 2016 [7]. However, the overall response rate to carfilzomib in the pivotal phase 2 clinical trial was less than $25 \%$ [8]. Furthermore, in a subsequent phase 3 study - in which overall survival was the primary endpoint [9] - carfilzomib monotherapy failed to significantly improve the survival of relapsed/refractory MM patients compared to those who received best supportive care (10.2 months vs 10.0 months) (ClinicalTrials.gov Identifier: 
NCT01302392). These results indicate that the majority of MM cells that became resistant to bortezomib were also resistant to carfilzomib. Clearly, to extend the life expectancy of patients with this disease, it is essential to characterize the mechanisms conferring resistance to proteasome inhibitors.

To begin to understand the underlying processes that might be relevant to clinical carfilzomib resistance in MM, we previously established carfilzomib-resistant derivatives of MM cell lines, KMS-11/Cfz and KMS-34/Cfz [10, 11]. In both cases, prosurvival autophagy was shown to contribute to carfilzomib resistance mediated, in part, via transcriptional upregulation of the SQSTM1 gene encoding sequestosome 1/p62 (SQSTM1/p62) [11]. SQSTM1/p62 is a multifunctional scaffold protein that interacts with various signaling molecules and serves as a ubiquitinbinding cargo receptor connecting the proteasomal and autophagic protein degradation pathways [12]. Another important function of SQSTM1/p62 is activation of the transcription factor nuclear factor-erythroid 2 (NF-E2)related factor 2 (Nrf2; gene symbol NFE2L2) in the Keap1-Nrf2 signaling pathway [13, 14]. The Keap1Nrf2 pathway maintains cellular redox homeostasis by inducing antioxidant and detoxification genes and by modulating energy metabolism [15]. A growing body of evidence implicates activation of the Keap1-Nrf2 pathway as a contributor to therapy resistance [16] but, to our knowledge, its role in conferring resistance to carfilzomib in $\mathrm{MM}$ cells has not been described.

The Keap1-Nrf2 pathway is tightly regulated at multiple levels, the precise details of which are currently being elucidated [17]. Under basal conditions, Nrf2 monomers are sequestered in cytoplasmic complexes through two-site binding to Keap1 homodimers which target Nrf2 for continuous ubiquitination and proteasomal degradation [18]. In response to oxidative stress, the Keap1 molecules are modified such that ubiquitination of Nrf2 is inhibited and it remains bound to the complex $[19,20]$. In a variation on this theme, overexpressed SQSTM1/p62 binds to and competitively prevents Keap 1 from interacting with one of the Nrf2 sites, effecting a conformational change in the complex which leads to impaired ubiquitination of associated $\mathrm{Nrf2}[13,14]$. In both scenarios, newly synthesized Nrf2 escapes cytoplasmic sequestration by Keap1 and is free to translocate to the nucleus and regulate gene expression. Of relevance in this regard, a number of reports have indicated that $\mathrm{Nrf2}$ expression is subject to diverse mechanisms of translational control [21-25].

Nrf2 activity can also be modulated by other mechanisms [26-29]. For example, nuclear translocation and activation of Nrf2 is enhanced by phosphorylation by the PERK protein kinase [26, 27]. PERK is activated upon accumulation of misfolded/unfolded proteins in the endoplasmic reticulum which results in the induction of an "unfolded protein response" (UPR) [30]. The best characterized function of PERK on UPR induction is to provide a protective advantage to the cell by attenuating global protein translation via inhibitory phosphorylation of eukaryotic translation initiation factor- $2 \alpha$ (eIF2 $\alpha)$ [31, 32]. Phosphorylation of eIF $2 \alpha$ also results in the preferential translation of certain mRNAs containing upstream open reading frames (uORFs), the prototypical example of which is activating transcription factor 4 (ATF4) [33]. During this phase of the UPR, ATF4 and Nrf2 coregulate transcription of some cytoprotective genes [34, 35]. However, if proteostasis is not restored, ATF4 induces a cell death program involving the CCAAT/enhancerbinding protein homologous protein (CHOP) transcription factor [36].

Here we report the establishment of a new carfilzomib-resistant derivative of the LP-1 MM cell line, LP-1/Cfz, in which carfilzomib resistance was characterized by induction of prosurvival autophagy as well as Nrf2 pathway activation associated with elevated SQSTM1/p62 levels. Unlike carfilzomib-resistant KMS11/Cfz and KMS-34/Cfz cells, increased SQSTM1/p62 levels were not due to transcriptional upregulation of the SQSTM1 gene [11]. Rather, the higher levels of SQSTM1/ p62 were due to increased translation dependent in part on activation of the PERK-eIF2 $\alpha$ axis. Comparative analysis with KMS-11/Cfz cells revealed Nrf2 target gene induction as well but only LP-1/Cfz cells were sensitized to carfilzomib by inhibition of the PERK-eIF2 $\alpha$ signaling cascade. Additionally, LP-1/Cfz cells exhibited increased Nrf2 synthesis associated with elevated expression of Nrf2 targets involved in translation initiation, in particular, EIF4E3 encoding an atypical eukaryotic translation initiation factor family member recently demonstrated to mediate context-specific translation in diffuse large B-cell lymphoma [37, 38]. Moreover, gene set enrichment analysis (GSEA) of gene expression profiling data from MM patient samples showed that increased EIF4E3 expression was predictive of Nrf2 activation in some chemoresistant minimal residual disease and relapsed/ refractory MM cases. These findings have elucidated further complexities of the proteostasis network in MM cells and provide preclinical rationale for therapeutic development of SQSTM1/p62-Nrf2 inhibitors as a means to overcome proteasome inhibitor resistance in a subgroup of advanced stage MM patients.

\section{RESULTS}

\section{GSEA identifies Nrf2 pathway activation in carfilzomib-resistant MM cell lines}

The carfilzomib-resistant LP-1/Cfz cell line was established by continuous culture of the LP-1 MM cell line [39] in stepwise increasing concentrations of the 
A

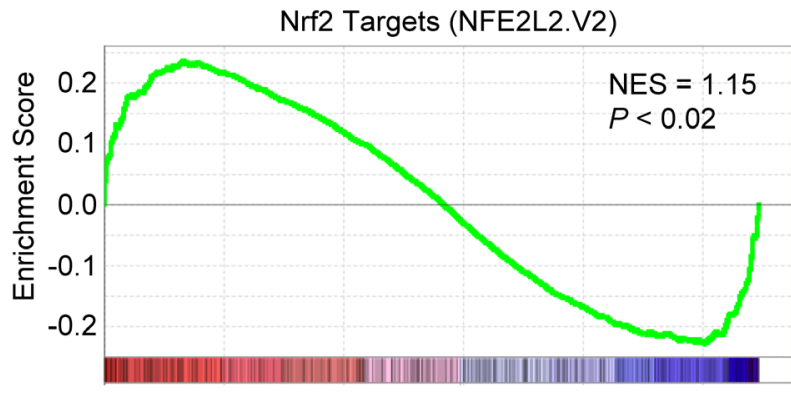

Up in LP-1/Cfz
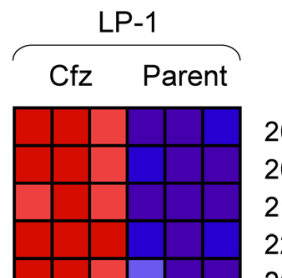

201141 at

209921 at SLC7A11 *

217678 at SLC7A11 *

222777_s_at WHSC1

205943_at TDO2

222778 s at WHSC1

205659_at HDAC9

204975_at EMP2

223472_at WHSC1

205413 at MPPED2

225079_at EMP2

203786_s_at TPD52L1

1564459 at DDC-AS1

219270 at $\mathrm{CHAC}$ *

47069_at PRR5

235903_at ANKS6

225078_at EMP2

219168_s_at PRR5

59697_at RAB15

214430_at GLA

$215692 \mathrm{~s}$ at MPPED2

$226193 \times$ at CBWD1

209053_s_at WHSC1

1557780_at WHSC1

218101 s at NDUFC2

223744_s_at SIAE

239521_at MFI2-AS1

211836_s_at MOG

213672 at MARS *

221810_at RAB15

210724_at EMR3

224839 s_at GPT2 *

213671 s at MARS *

209052_s_at WHSC1

242311_x_at WHSC1

208373 s_at P2RY6

202504 at TRIM29

235911_at MFI2

209054_s_at WHSC1

212441 at
B

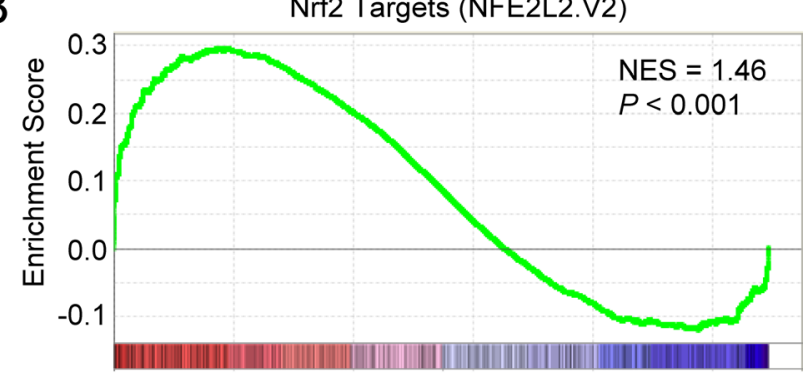

Up in KMS-11/Cfz

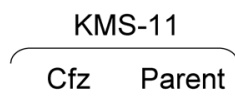

201463_s_at TALDO1*

206435_at B4GALNT1

213112 s at SQSTM1 *

224954_at SHMT1

225252_at SRXN1*

207469_s_at PIR

214430_at GLA

203427_at ASF1A

203428_s_at ASF1A

203814_s_at NQO2

206395_at DGKG

244804_at SQSTM1*

201471_s_at SQSTM1*

219475 at OSGIN1

206742_at FIGF

209448_at HTATIP2

210519_s_at NQO1*

201467 s at NQO1*

1555372_at BCL2L11

1565228_s_at ALB

206153 at CYP4F11

212526 at SPG20

1555385_at B4GALNT1

242195_x_at NUMBL

207180 s at HTATIP2

224176_s_at AXIN2

201468_s_at NQO1*

225609_at GSR *

201266_at TXNRD1*

218581_at ABHD4

203665_at $\mathrm{HMOX} 1 *$

203473_at SLCO2B1

226208 at ZSWIM6

231702_at TDO2

1553418_a_at CNTNAP5

239067_s_at PANX2

205645 at REPS2

205943_at TDO2

236140_at GCLM *

240223 at SLC2A9

Figure 1: Different sets of Nrf2 target genes are upregulated in LP-1/Cfz and KMS-11/Cfz cells. A. GSEA enrichment plot and heat map of the leading edge subset of Nrf2 target genes upregulated in LP-1/Cfz (Cfz) versus parental LP-1 (Parent) cells (triplicate samples). Selected Nrf2 targets that are coregulated by ATF4 are indicated with asterisks. B. GSEA enrichment plot and heat map of the leading edge subset of Nrf2 target genes upregulated in KMS-11/Cfz (Cfz) versus parental KMS-11 (Parent) cells (triplicate samples). Prototypical Nrf2 targets are indicated with asterisks. Probe sets for the SQSTM1 gene are highlighted. Some Nrf2 targets (e.g., TDO2 and GLA) were upregulated in both LP-1/Cfz and in KMS-11/Cfz cells. Gene set: NFE2L2.V2 (M2870). NES, normalized enrichment score. 
Table 1: Overrepresented transcription factor binding site (TFBS) motifs in genes with increased expression in LP-1/ Cfz and KMS-11/Cfz cells*

\section{A. LP-1/Cfz cells}

\begin{tabular}{|l|l|l|l|l|}
\hline TFBS & JASPAR ID & Class & Family & Fisher Score \\
\hline Arnt::Ahr & MA0006.1 & Zipper-Type & Helix-Loop-Helix & 27.7 \\
\hline Hand1::Tcfe2a & MA0092.1 & Zipper-Type & Helix-Loop-Helix & 26.2 \\
\hline SP1 & MA0079.2 & Zinc-coordinating & BetaBetaAlpha-zinc finger & 24.3 \\
\hline AP1 & MA0099.2 & Zipper-Type & Leucine Zipper & 24.1 \\
\hline Nkx3-2 & MA0122.1 & Helix-Turn-Helix & Homeo & 24.0 \\
\hline MEF2A & MA0052.1 & Other Alpha-Helix & MADS & 23.4 \\
\hline TBP & MA0108.2 & Beta-sheet & TATA-binding & 23.0 \\
\hline NFATC2 & MA0152.1 & Ig-fold & Rel & 21.9 \\
\hline MZF1_5-13 & MA0057.1 & Zinc-coordinating & BetaBetaAlpha-zinc finger & 21.8 \\
\hline NFE2L2 & MA0150.1 & Zipper-Type & Leucine Zipper & 21.4 \\
\hline
\end{tabular}

B. KMS-11/Cfz cells

\begin{tabular}{|l|l|l|l|l|}
\hline TFBS & JASPAR ID & Class & Family & Fisher Score \\
\hline NFE2L2 & MA0150.1 & Zipper-Type & Leucine Zipper & 22.7 \\
\hline NFYA & MA0060.1 & Other Alpha-Helix & NFY CCAAT-binding & 17.3 \\
\hline Spz1 & MA0111.1 & Other & Other & 14.2 \\
\hline Foxa2 & MA0047.2 & Winged Helix-Turn-Helix & Forkhead & 13.4 \\
\hline INSM1 & MA0155.1 & Zinc-coordinating & BetaBetaAlpha-zinc finger & 13.2 \\
\hline RELA & MA0107.1 & Ig-fold & Rel & 13.0 \\
\hline REL & MA0101.1 & Ig-fold & Rel & 12.7 \\
\hline Esrrb & MA0141.1 & Zinc-coordinating & Hormone-nuclear Receptor & 12.5 \\
\hline FEV & MA0156.1 & Winged Helix-Turn-Helix & Ets & 12.5 \\
\hline CEBPA & MA0102.2 & Zipper-Type & Leucine Zipper & 12.4 \\
\hline
\end{tabular}

*Identified by oPOSSUM-3 Single Site Analysis using JASPAR CORE vertebrate profiles (minimum information content = 8 bits; matrix score threshold: $85 \%$ ) and ranked by Fisher score. Search regions: 5,000 bp upstream and 5,000 bp downstream of transcription start sites. The NFE2L2 binding site motif was identified in both LP-1/Cfz and KMS-11/Cfz upregulated gene sets. Fisher scores reflect the number of genes containing the predicted TFBS.

drug ( $4 \mathrm{nM}$ to $12 \mathrm{nM}$ ) over an 18 week period according to a previously published protocol used to derive the carfilzomib-resistant MM cell lines, KMS-11/Cfz and KMS-34/Cfz [11] (Figure S1). As observed for KMS11/Cfz and KMS-34/Cfz cells, LP-1/Cfz cells retained resistance to carfilzomib even when tested after removal of selective pressure for approximately 8 weeks. Gene expression profiling was performed on LP-1/Cfz and parental LP-1 cells, and GSEA was used to identify differentially overrepresented pathways and processes associated with carfilzomib resistance shared between LP-1/Cfz cells and the KMS-11/Cfz and KMS-34/Cfz cell lines [11].

We first applied GSEA to examine gene sets from the C3:TFT (transcription factor targets) subcollection of the Molecular Signatures Database (MSigDB). Binding site motifs for the "cap ' $n$ ' collar" transcription factors NF-E2 (V\$NFE2_01) and Nrf2 (V\$NRF2_Q4) that recognize a similar AP1-like core consensus sequence (TGA(G/C/T)TCA) [40] were significantly enriched in genes with increased expression in LP-1/Cfz and KMS$11 / \mathrm{Cfz}$ cells (Figure S2). Although NF-E2 expression is restricted to erythroid cells, Nrf2 is broadly expressed and activated in response to stress [41, 42]. In line with Nrf2 activation in both carfilzomib-resistant MM models, significant enrichment of Nrf2 target genes (NFE2L2.V2 gene set) was observed when GSEA was applied to the C6 (oncogenic signatures) collection of MSigDB (Figure 1).

GSEA detects coordinated expression changes in groups of genes by taking all genes into account, even if individual representatives of the groups exhibit modest changes [43]. In a second, complementary approach, we employed fold change (FC) cutoffs to compare similar numbers of differentially expressed genes in LP-1/Cfz (FC $\geq 1.7 ; 896$ probe sets) and KMS-11/Cfz cells (FC $\geq 1.4 ; 887$ probe sets). Transcription factor binding site 
motif discovery was carried out using the oPOSSUM-3 Single Site Analysis tool and the JASPAR CORE vertebrate database [44]. NFE2L2 was the highestscoring motif in KMS-11/Cfz cells and the only motif in common among the top ten ranked transcription factor binding sites overrepresented in the promoter regions of the upregulated genes in both carfilzomib-resistant MM models (Table 1). This result was notable because an Nrf2 ChIP-seq dataset was one of the data sources used to validate the performance of the oPOSSUM-3 program [41, 44]. The Nrf2 ChIP-seq dataset comprised basal $\left(\mathrm{Nrf2}^{-/-}\right)$ and inducible $\left(\right.$ Keap $\left.^{-/}\right)$direct binding targets of Nrf2 in mouse embryo fibroblasts [41]. We combined this dataset with Nrf2 direct binding targets identified by ChiP-seq experiments in human lymphoblastoid cells after treatment with the dietary isothiocyanate, sulforaphane [40]. Using GeneSpring analysis software, we found significant enrichment of these $\mathrm{Nrf} 2$ direct binding targets in the differentially expressed genes in LP-1/Cfz cells (102 out of 896 probe sets; $\left.P<10^{-31}\right)$ and KMS-11/Cfz cells (107 out of 887 probe sets; $P<10^{-31}$ ) (see Table S1 for the probe set lists). Moreover, there was also significant overlap of the differentially expressed genes in the carfilzomibresistant MM cells and genes that were downregulated in human lymphoblastoid cells after sulforaphane treatment — LP-1/Cfz cells (72 out of 896 probe sets; $P<10^{-20}$ ) and KMS-11/Cfz cells ( 68 out of 887 probe sets; $P<10^{-20}$ ) which may represent indirect Nrf2 targets (see Table S2 for the probe set lists).

Strikingly, different Nrf2 target genes were upregulated in LP-1/Cfz and KMS-11/Cfz cells. Prototypical cytoprotective Nrf2 targets, as exemplified by NQO1 [15], were significantly upregulated in KMS11/ Cfz cells [11] but not in LP-1/Cfz cells (Figure 1; Table $\mathrm{S} 1)$. These findings suggested differential levels of Nrf2 activation and/or that the Nrf2-interacting partners might be different in the two carfilzomib-resistant MM model systems. In support of the latter possibility, GSEA indicated activation of a prosurvival ATF4 target gene response in LP-1/Cfz cells (Figure S3A, S3B). GSEA also showed enrichment of HER2/ERBB2-related signatures in LP-1/Cfz cells when the C2:CP (canonical pathways) subcollection of MSigDB was queried (Figure $\mathrm{S} 3 \mathrm{C}, \mathrm{S} 3 \mathrm{D})$. Both factors were previously reported to cooperate with $\mathrm{Nrf} 2$ and modulate specificity of $\mathrm{Nrf} 2$ target gene activation [35, 36, 45, 46]. In particular, a number of the Nrf2 targets upregulated in LP-1/Cfz cells (indicated in Figure 1A) were previously shown to be directly coregulated by ATF4 [34, 47]. Regardless of the different transcriptional outcomes, taken together, the data implicated activation of Nrf2 pathways in the acquisition of carfilzomib resistance in both MM models. In the following experiments, we concentrated mainly on elucidating the molecular mechanisms and potential functional significance of $\mathrm{Nrf} 2$ pathway activation in LP-1/Cfz cells.

\section{The autophagy-related gene $G A B A R A P L 1$ is an Nrf2 target upregulated in carfilzomib-resistant MM cells}

We verified the microarray data for selected $\mathrm{Nrf} 2$ targets in LP-1/Cfz cells by real-time reverse transcription polymerase chain reaction (qRT-PCR) assay and western blot analysis (Figure S4; see also Figure 2C and Figure 9C). We noted that several of the novel Nrf2 targets are involved in translational control mechanisms; for example, EEF1A2, EIF4E3, RND3/RhoE (Table S1A) and FAM129A/Niban (Table S2A). EEF1A2 is a translation elongation factor gene that was previously shown to promote survival of mouse plasmacytoma cells [48], whereas EIF4E3, RND3/RhoE and FAM129A/Niban encode proteins that participate in various facets of capdependent translation initiation (described in more detail below) $[38,49,50]$.

Of the validated genes, we focused initially on the autophagy-related gene GABARAPL1 [51] since it was also significantly upregulated in KMS-11/Cfz cells (Table S1B). Indeed, inspection of the enriched V\$NFE2_01 transcription factor motif gene set indicated that GABARAPL1 was the top-ranked upregulated Nrf2 target in common between LP-1/Cfz and KMS-11/ Cfz cells (Figure 2A). In this regard, it was noteworthy that the KEGG autophagy gene set was among the significantly enriched gene sets in the MSigDB C2:CP (canonical pathways) collection identified by combined pairwise comparison of LP-1/Cfz and KMS-11/Cfz versus parental LP-1 and KMS-11 cells, and GABARAPL1 was the top-ranked gene in this instance as well (Figure 2B). To investigate whether GABARAPL1 is an Nrf2-binding target in LP-1/Cfz and KMS-11/Cfz cells, we performed ChIP-qPCR analysis on the promoter region of the GABARAPL1 gene.

An evolutionarily conserved Nrf2 motif was identified in the GABARAPL1 promoter region by the ConTra v2 transcription factor binding site motif discovery program using position weight matrices from both the JASPAR CORE and TRANSFAC database libraries [52] (Figure S5A). Of note, this motif coincided with an NF-E2 ChIP-seq binding site identified in K562 erythroid cells by the ENCODE project (Figure S5B). Using primers flanking this site, we found specific enrichment of Nrf2-precipitated chromatin in comparison to the unrelated antibody control precipitation in both MM cell lines, increased binding of which was observed in the carfilzomib-resistant derivatives (Figure 2D). This result is congruent with previous findings of substantial overlap of functional Nrf2 and NF-E2 binding sites at the genomewide and individual gene levels $[40,42]$. Thus, the data support the notion that GABARAPL1 is a direct Nrf2 target gene in LP-1/Cfz and KMS-11/Cfz cells.

GABARAPL1 is involved in selective autophagy through its interaction with ubiquitin-binding cargo 
A

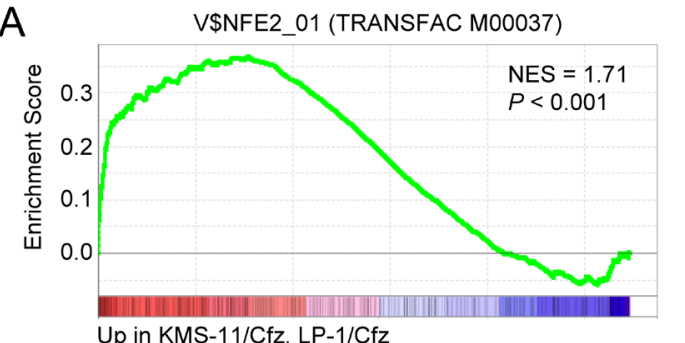

Up in KMS-11/Cfz, LP-1/Cfz
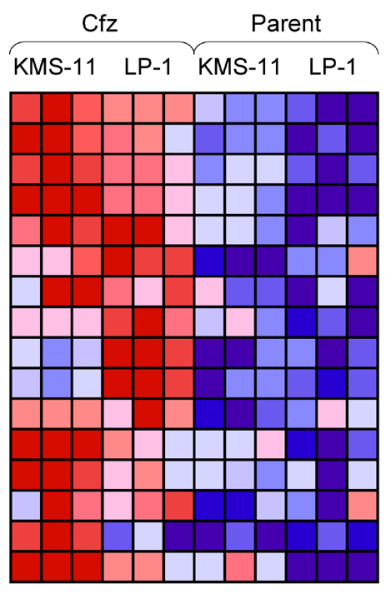

208869_s_at GABARAPL1

208868_s_at GABARAPL1

$217007 \mathrm{~s}$ at ADAM15

201215_at PLS3

238994_at OTUD7B

$205142 \times$ at ABCD1

1556831_at DYNC1H1

218111_s_at CMAS

217398_x_at GAPDH

212581_x at GAPDH

237626 at RB1CC1

1555896_a_at ADAM15

203348 s at ETV5

204268_at S100A2

244804_at SQSTM1

217432_s_at IDS

C

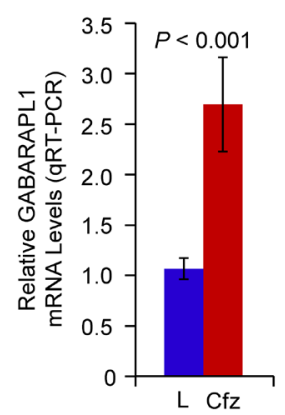

E

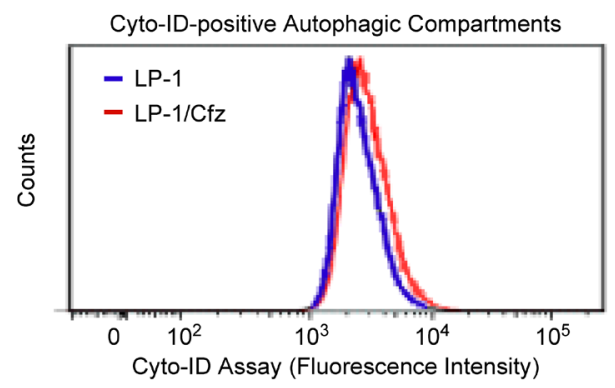

B

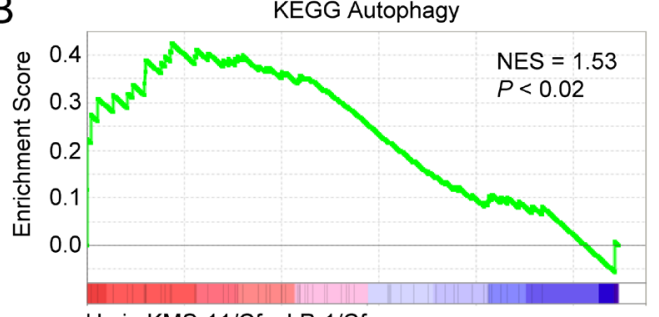

Up in KMS-11/Cfz, LP-1/Cfz
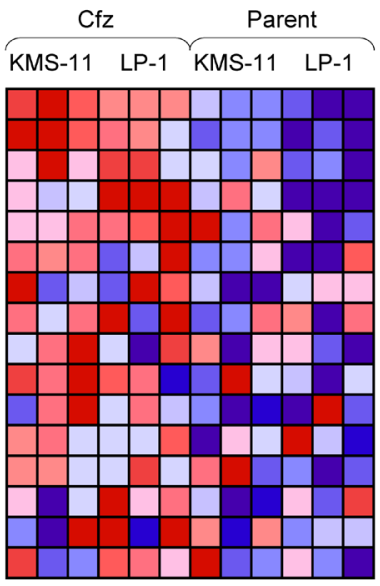

208869_s_at GABARAPL1 208868_s_at GABARAPL1 207932 at IFNA8 213115_at ATG4A 211145_x_at IFNA21 234080 at ATG5 211338_at IFNA2 207709_at PRKAA2 210354_at IFNG 204297_at PIK3C3 216436 at PIK3R4 216752 at PIK3R4 1556240 at ATG7 1569272_at PIK3C3 $207964 x$ at IFNA4 211405_x_at IFNA17

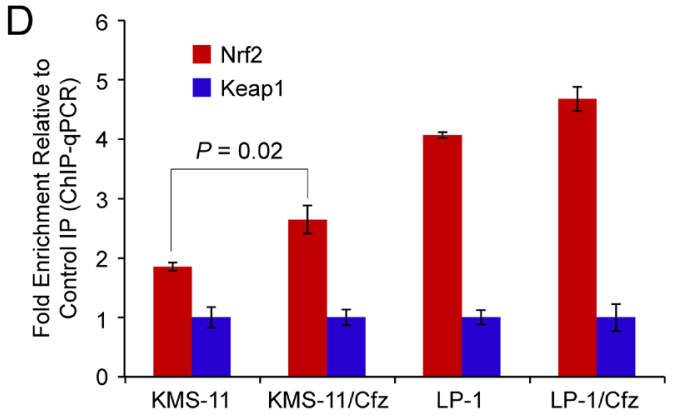

F

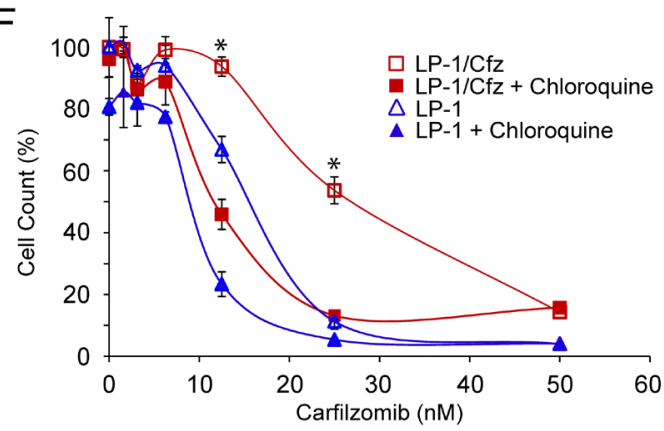

Figure 2: The autophagy-related gene GABARAPL1 is an Nrf2 binding target upregulated in LP-1/Cfz and KMS-11/ Cfz cells. A. GSEA enrichment plot and heat map of the leading edge subset of genes upregulated in both LP-1/Cfz and KMS-11/Cfz cells (triplicate samples) whose promoter regions contain the NF-E2 motif. Gene set: V\$NFE2_01 (M1608). The top-ranked probe sets corresponded to GABARAPL1. B. GSEA enrichment plot and heat map of the leading edge subset of autophagy pathway genes upregulated in both LP-1/Cfz and KMS-11/Cfz cells. The top-ranked probe sets corresponded to GABARAPL1. Gene set: KEGG Regulation of autophagy (M6382; KEGG Pathway hsa04140). C. qRT-PCR analysis was performed to validate the differential expression of GABARAPL1 mRNA in LP-1/Cfz (Cfz) versus parental LP-1 (L) cells (mean values of three qRT-PCR experiments). See Table S1A for expression changes determined from the microarray data. D. Increased binding of Nrf2 to the GABARAPL1 promoter region indicated in Figure S5 in LP-1/ Cfz and KMS-11/Cfz cells as determined by ChIP-qPCR. E. Fluorescence histograms of LP-1/Cfz and parental LP-1 cells stained with the Cyto-ID autophagy detection reagent. F. Cells were treated with the indicated concentrations of carfilzomib for 72 hours in the absence or presence of chloroquine $(10 \mu \mathrm{M})$ and cell viability was determined by alamarBlue assay. ${ }^{*}, P<0.001 v$ s carfilzomib alone $(n=3)$. 
receptors such as SQSTM1/p62 [53] and it is essential during the late stages of autophagosome maturation [54]. Previously, we showed that increased autophagic flux contributes to carfilzomib resistance in KMS-11/Cfz cells [11]. Increased staining intensity with the Cyto-ID autophagy detection reagent revealed that LP-1/CfZ cells also had higher steady state levels of autophagosomes
(Figure 2E), indicating that activation of the Nrf2GABARAPL1 axis correlates with increased autophagic activity [55]. We also previously demonstrated that inhibition of autophagy by chloroquine treatment, which increases lysosomal $\mathrm{pH}$ and blocks autophagosomelysosome fusion, sensitized $\mathrm{KMS}-11 / \mathrm{Cfz}$ cells to carfilzomib [11]. Cotreatment with chloroquine likewise
A

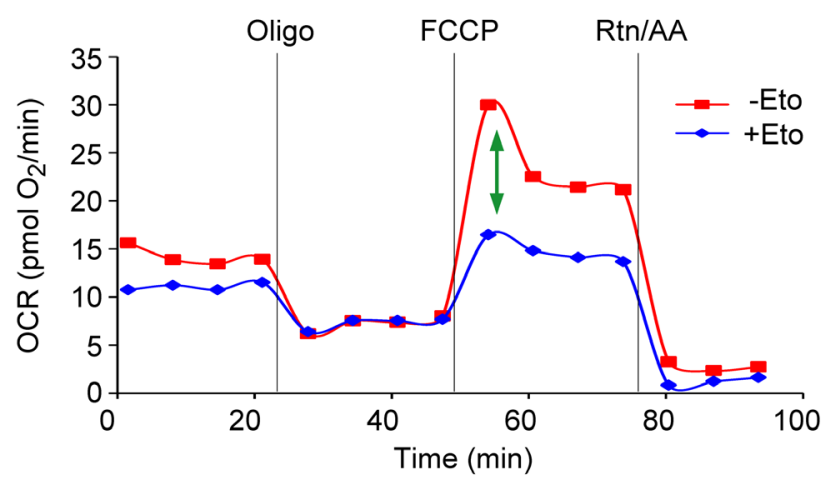

Fatty Acid Oxidation (LP-1)

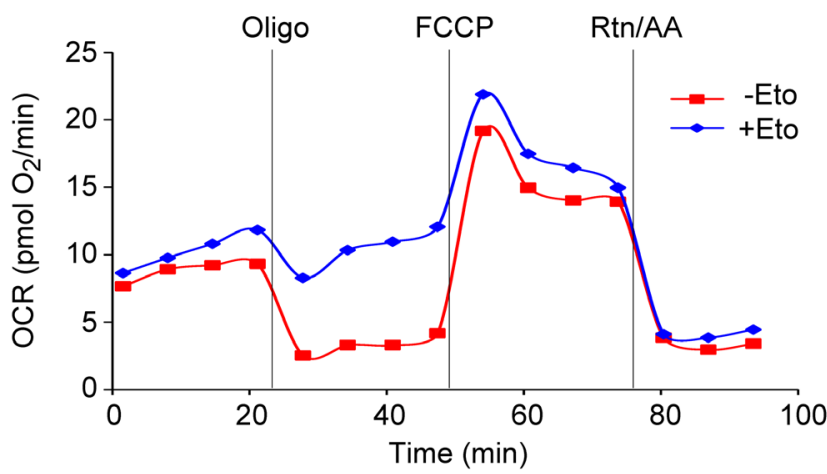

B

Increased Fatty Acid Oxidation (LP-1/Cfz)

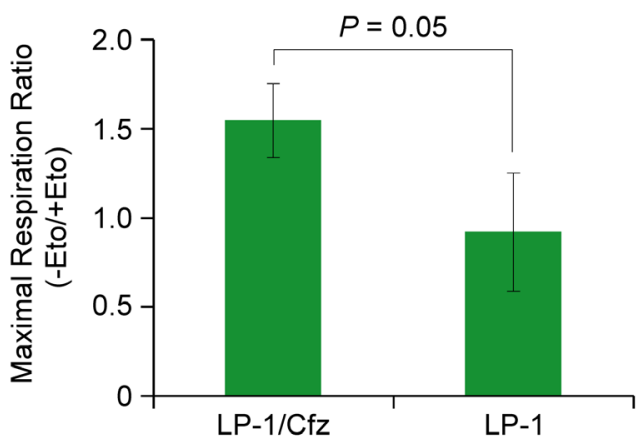

C

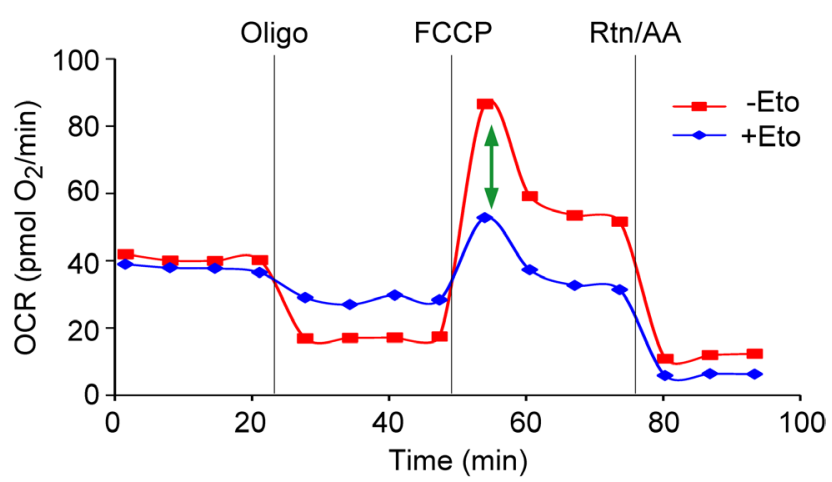

Fatty Acid Oxidation (KMS-11)

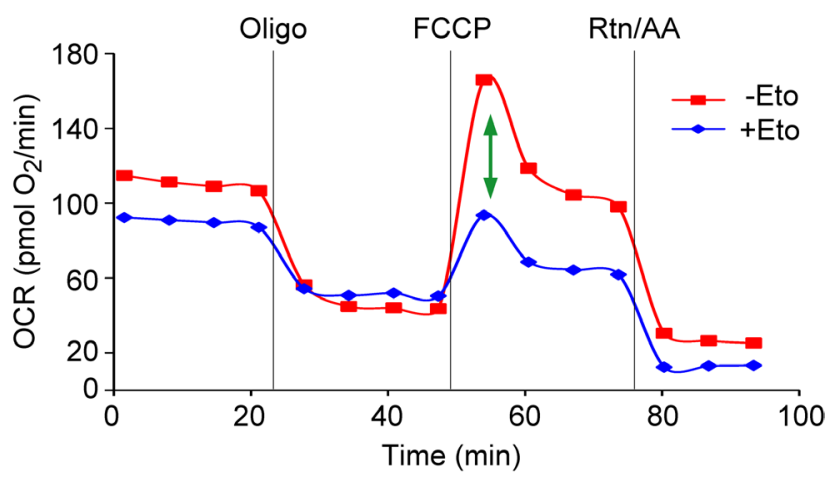

Do Change in Fatty Acid Oxidation (KMS-11/Cfz)

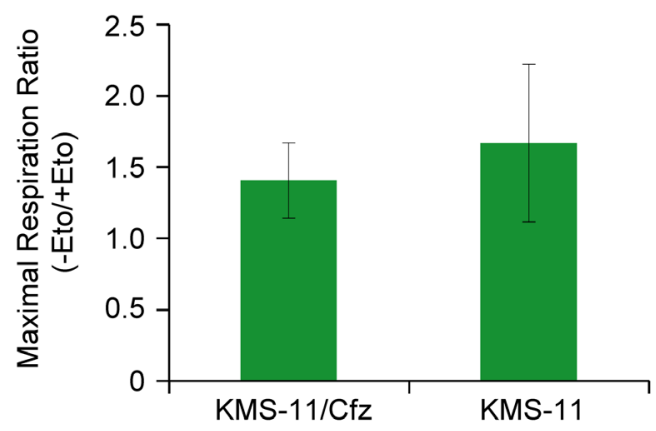

Figure 3: LP-1/Cfz cells exhibit increased FAO. A. Oxygen consumption rate (OCR) was measured using the Seahorse XF24 Extracellular Flux Analyzer. FAO was determined by XF Cell Mito Stress Test using the XF Palmitate-BSA FAO substrate in the absence or presence of etomoxir (Eto), an inhibitor of carnitine palmitoyltransferase-1, the rate limiting enzyme in FAO. The ATP synthase inhibitor oligomycin (Oligo), the uncoupler carbonyl cyanide 4-(trifluoromethoxy) phenylhydrazone (FCCP), and the complex I and III inhibitors rotenone and antimycin $\mathrm{A}(\mathrm{Rtn} / \mathrm{AA})$ were injected at the indicated times. The green arrow indicates the difference in maximal respiration — due to FAO - when LP-1/Cfz cells were treated with FCCP in the absence or presence of Eto. B. Average increase in FAO in LP-1/Cfz versus parental LP-1 cells $(n=3)$. C. Oxidation of fatty acids was determined in KMS-11/Cfz and KMS-11 cells as described in A. D. No change in FAO in KMS-11/Cfz versus parental KMS-11 cells $(n=3)$. 
diminished carfilzomib resistance in LP-1/Cfz cells (Figure 2F), indicating that prosurvival autophagy contributes to acquired carfilzomib resistance in this MM cell line similarly to KMS-11/Cfz cells.

\section{LP-1/Cfz cells exhibit increased antioxidant capacity due to altered intermediary metabolism}

As mentioned above, Nrf2 targets directly involved in reactive oxygen species (ROS) detoxification were not significantly upregulated in LP-1/Cfz cells by comparison to parental LP-1 cells. However, examination of the microarray data revealed that a number of these prototypical Nrf2 target genes (including NQO1, GCLC, GCLM, GPX4, GSR, GSTM1) are already highly expressed in LP-1 cells. NQO1 ((NAD(P)H:quinone oxidoreductase

A
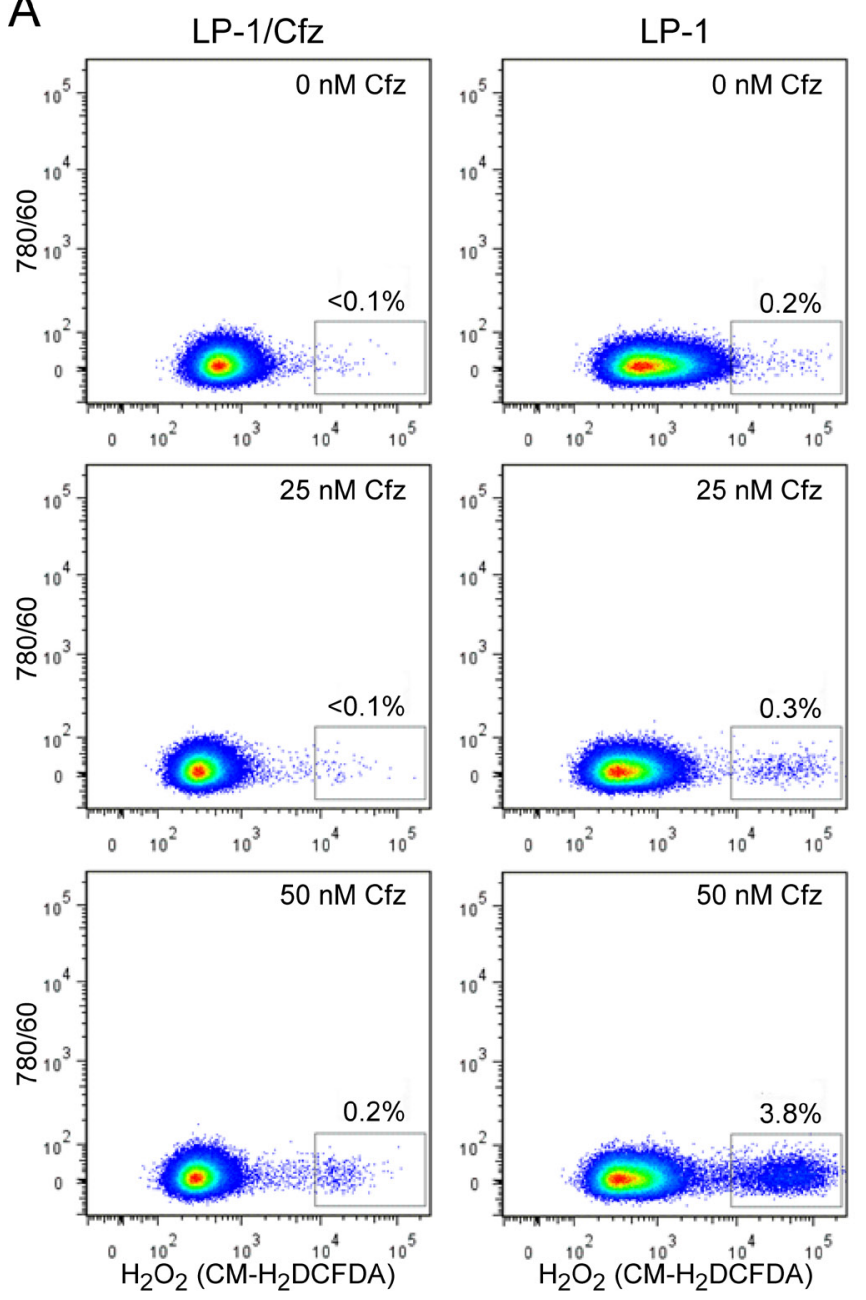

1), GSR (glutathione reductase) and other antioxidantassociated enzymes regulated by Nrf2 require NADPH as a reducing cofactor [15]. Accordingly, Nrf2 also regulates several NADPH-generating enzymes. Specifically, Nrf2 facilitates NADPH production by directing carbon flux through the pentose phosphate pathway [56]. Consistent with the hypothesis that $\mathrm{Nrf} 2$ is activated in LP-1/Cfz as well as in KMS-11/Cfz cells, NADPH levels were increased concomitant with upregulation of KEGG pentose phosphate pathway genes in both models (Figure S6).

It is becoming increasingly appreciated that Nrf2 also affects multiple aspects of intermediary metabolism indirectly [15], including mitochondrial fatty acid oxidation (FAO) [57]. Of note, during the metabolic shift towards FAO, activation of autophagy hydrolyzes lipids into fatty acids for fuel [58]. Considering that FAO is

B
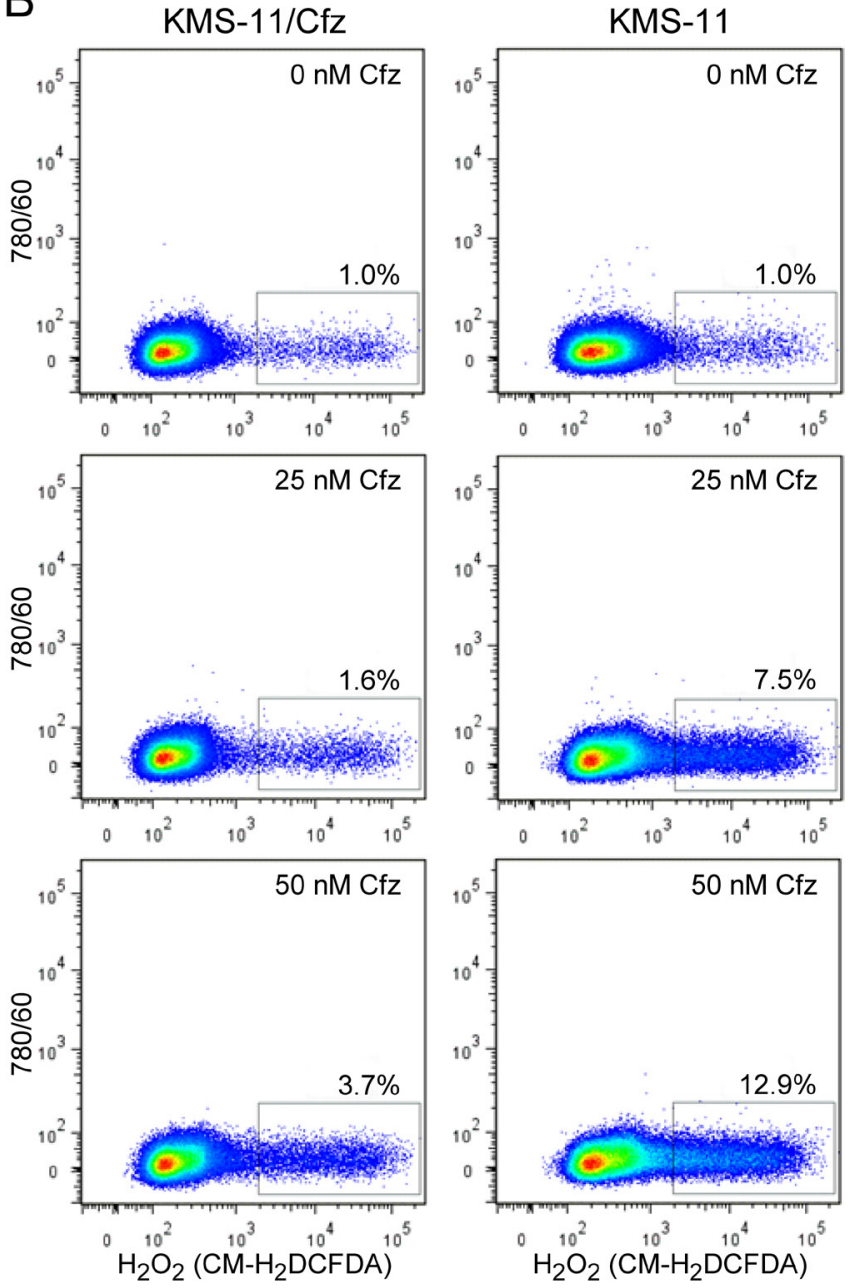

Figure 4: LP-1/Cfz and KMS-11/Cfz cells contain lower levels of hydrogen peroxide-associated ROS than parental LP-1 and KMS-11 cells following carfilzomib treatment. A. LP-1/Cfz and parental LP-1 cells were treated for 18 hours with the indicated concentrations of carfilzomib $(\mathrm{Cfz})$ and cellular hydrogen peroxide $\left(\mathrm{H}_{2} \mathrm{O}_{2}\right)$ levels were quantified by flow cytometry using the redox-sensitive CM-H DCFDA fluorescent dye. B. KMS-11/Cfz and parental KMS-11 cells were treated for 18 hours with the indicated concentrations of carfilzomib and cellular hydrogen peroxide $\left(\mathrm{H}_{2} \mathrm{O}_{2}\right)$ levels were quantified by flow cytometry using the redox-sensitive CM- ${ }_{2}$ DCFDA fluorescent dye. 
an important source of NADPH in leukemic cells [59], we examined whether rates of FAO were increased in carfilzomib-resistant LP-1/Cfz and KMS-11/Cfz cells. Increased FAO was observed in LP-1/Cfz versus parental LP-1 cells (Figure 3A, 3B). By comparison, basal rates of FAO were already elevated in parental KMS-11 cells and there was no further increase in KMS-11/Cfz cells (Figure 3C, 3D).

Considered together, the gene expression profiling and functional assays supporting increased NADPH production and/or upregulation of genes that detoxify ROS suggested that LP-1/Cfz and KMS-11/Cfz cells would exhibit enhanced Nrf2-mediated antioxidant capacity. To examine this, we measured ROS generation using the fluorescent redox-sensitive dyes CM- $\mathrm{H}_{2}$ DCFDA, which is specific for hydrogen peroxide, and MitoSOX Red, which is selective for superoxide [60]. Cells were treated with varying concentrations of carfilzomib and ROS levels were quantified by flow cytometry. Lower levels of ROS were generated in LP-1 cells compared to KMS11 cells under all conditions; at the highest carfilzomib concentrations, both hydrogen peroxide (Figure 4) and superoxide (Figure 5) levels were reduced by more than $70 \%$ in the carfilzomib-resistant derivatives versus their parental counterparts.

To determine whether antioxidant defense conferred protection to carfilzomib in these MM models and whether there was a contribution of FAO, the cells were cotreated with carfilzomib and either (S)-4-carboxyphenylglycine, an inhibitor of $\mathrm{xCT}$ (a subunit of the $\mathrm{x}_{\mathrm{c}}^{-}$cystine antiporter involved in glutathione homeostasis encoded by the SLC7A11 gene; see Figure 1A) [34] or etomoxir, an inhibitor of carnitine palmitoyltransferase-1 (the rate limiting enzyme in FAO; see Figure 3) [61]. Cotreatment with either inhibitor resulted in enhanced sensitivity of the resistant cells to carfilzomib (Figure 6), indicating that
A
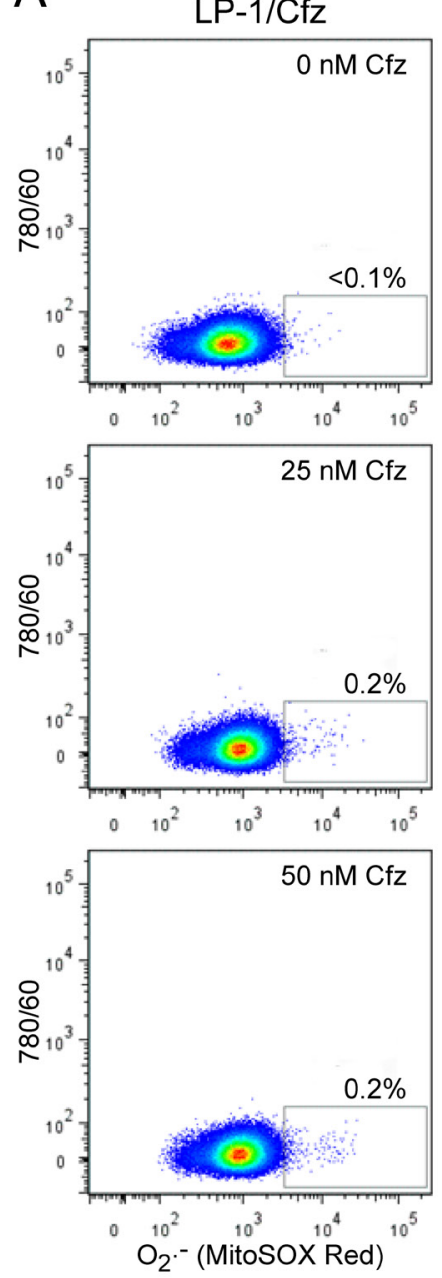

LP-1
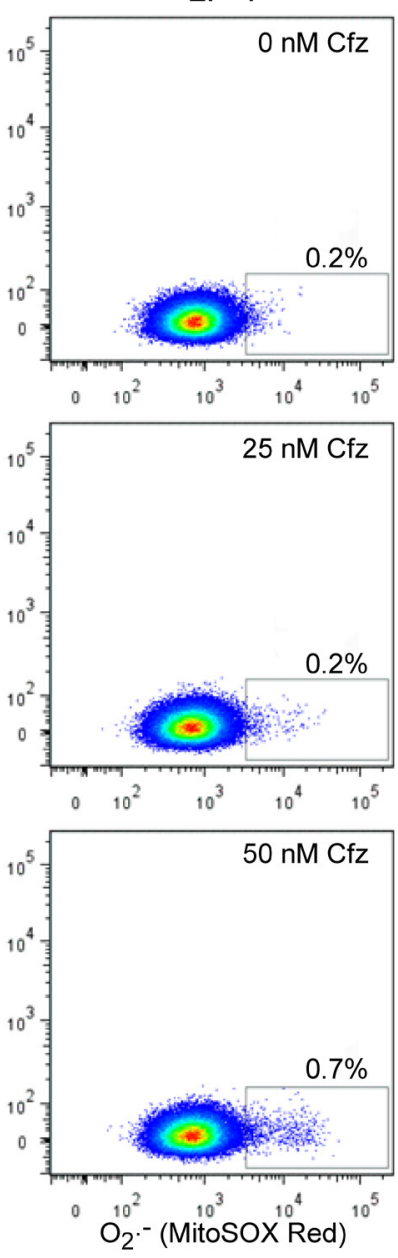

B
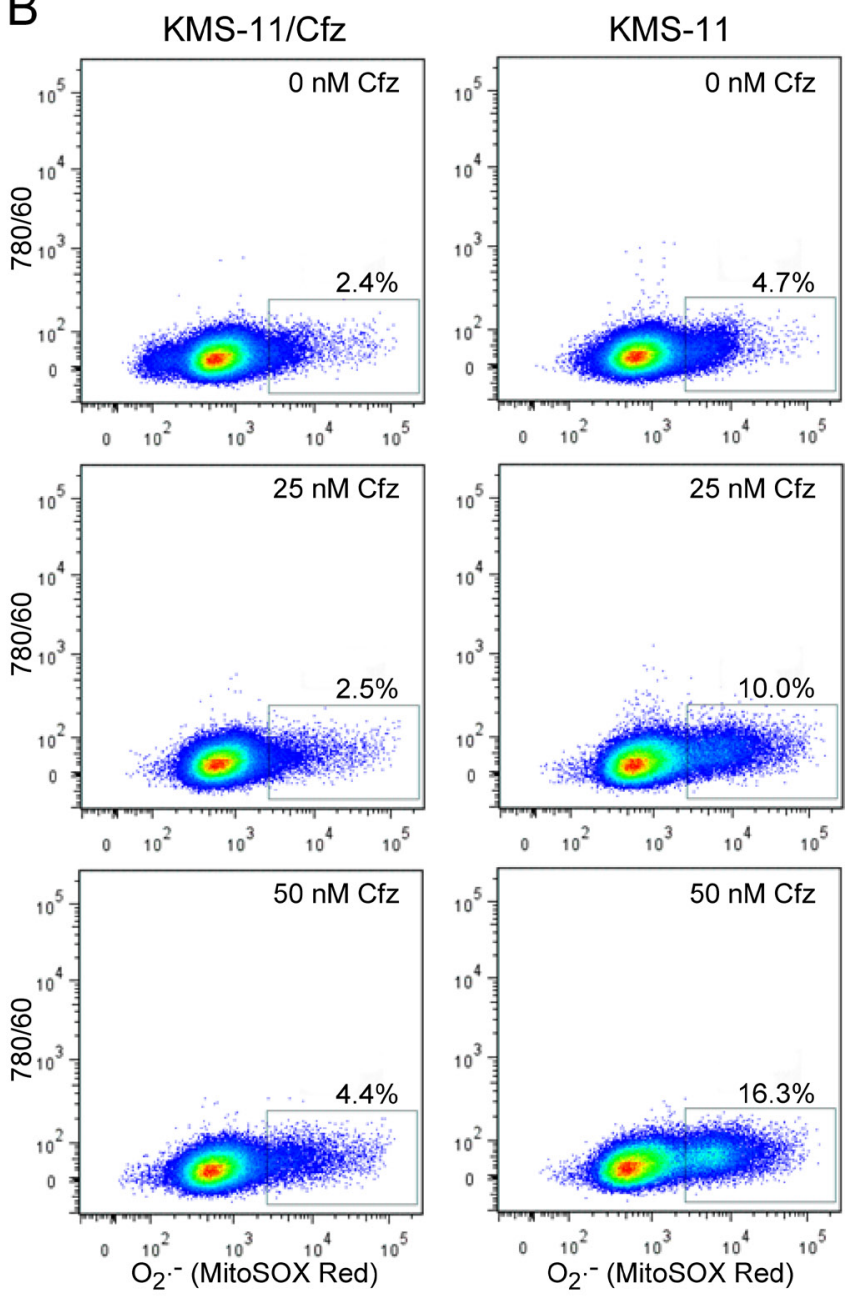

Figure 5: LP-1/Cfz and KMS-11/Cfz cells contain lower levels of superoxide than parental LP-1 and KMS-11 cells following carfilzomib treatment. A. LP-1/Cfz and parental LP-1 cells were treated for 18 hours with the indicated concentrations of carfilzomib $(\mathrm{Cfz})$ and superoxide $\left(\mathrm{O}_{2}^{-}\right)$levels were quantified by flow cytometry using the redox-sensitive MitoSOX Red fluorescent dye. B. KMS-11/Cfz and parental KMS-11 cells were treated for 18 hours with the indicated concentrations of carfilzomib and superoxide $\left(\mathrm{O}_{2}^{-}\right)$ levels were quantified by flow cytometry using the redox-sensitive MitoSOX Red fluorescent dye. 
the glutathione-based antioxidant system contributes to carfilzomib resistance in these MM models and that FAO is a potential source of reducing equivalents underlying the increased ROS defense.

Finally, to directly test whether Nrf2 activity contributes to carfilzomib resistance, we transfected LP-1/Cfz and parental LP-1 cells with two previously validated Nrf2 siRNAs and a control siRNA with no known mammalian homology $[62,63]$. We confirmed that Nrf2 mRNA was knocked down $\sim 45-65 \%$ by qRTPCR. This was accompanied by an $\sim 30-45 \%$ decrease in GABARAPL1 mRNA levels (Figure 7A). As illustrated in Figure 7B, both Nrf2 siRNAs sensitized LP-1/Cfz cells to carfilzomib.

\section{Activation of the PERK-eIF2 $\alpha$ axis contributes to SQSTM1/p62 synthesis and carfilzomib resistance in $\mathrm{LP}-1 / \mathrm{Cfz}$ cells}

Next, we focused on the molecular mechanisms underlying Nrf2 activation in LP-1/Cfz cells. Nrf2 mRNA levels were not significantly increased. However, western blot analyses showed significantly higher Nrf2 protein levels in the high salt (nuclear) fraction of LP-1/Cfz cell lysates compared to parental LP-1 cell lysates $(P<0.001)$ (Figure 7C). Immunofluorescence confocal microscopy confirmed that Nrf2 had a predominantly perinuclear and nuclear localization in LP-1/Cfz cells (Figure 7D)
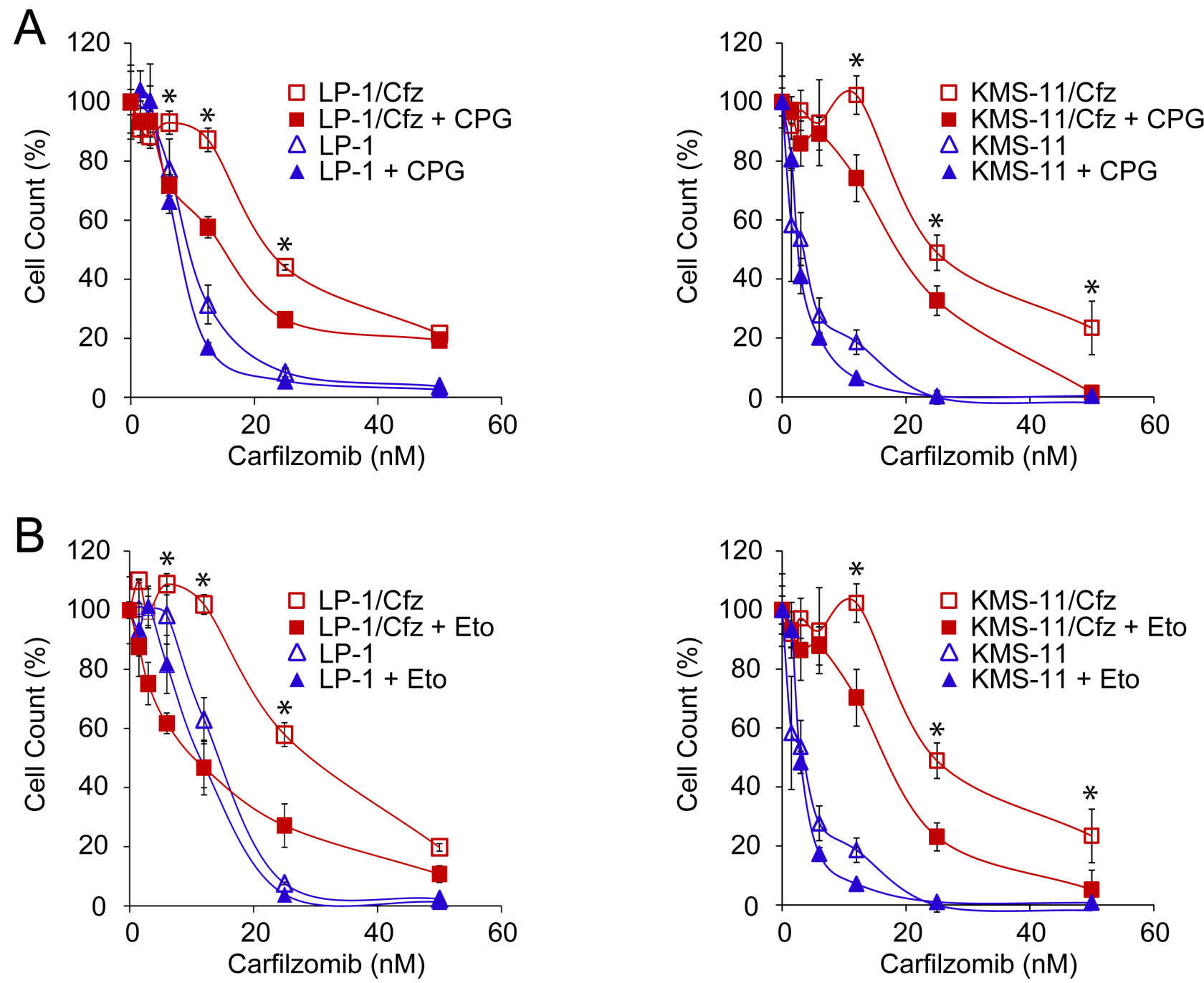

Figure 6: Inhibition of glutathione homeostasis or FAO sensitizes LP-1/Cfz and KMS-11/Cfz cells to carfilzomib. A. Treatment with (S)-4-carboxyphenylglycine (CPG), an inhibitor of xCT (a subunit of the $\mathrm{x}_{\mathrm{c}}^{-}$cystine antiporter involved in glutathione homeostasis encoded by the SLC7A11 gene) sensitizes LP-1/Cfz cells (left graph) and KMS-11/Cfz cells (right graph) to carfilzomib. Cells were treated with the indicated concentrations of carfilzomib for 72 hours in the absence or presence of CPG $(0.2 \mathrm{mM})$ and cell viability was determined by alamarBlue assay. ${ }^{*}, P<0.001 v s$ carfilzomib alone $(n=3)$. B. Treatment with etomoxir, an inhibitor of carnitine palmitoyltransferase-1 (the rate limiting enzyme in FAO), sensitizes LP-1/Cfz cells (left graph) and KMS-11/Cfz cells (right graph) to carfilzomib. Cells were treated with the indicated concentrations of carfilzomib for 72 hours in the absence or presence of etomoxir $(20 \mu \mathrm{M})$ and cell viability was determined by alamarBlue assay. ${ }^{*}, P<0.001 v s$ carfilzomib alone $(n=3)$. 
[64]. Moreover, treatment with trigonelline, an inhibitor of Nrf2 nuclear import [63], sensitized LP-1/Cfz cells to carfilzomib (Figure 7E). Steady state Keap1 levels were not reduced in LP-1/Cfz cells compared to parental LP-1 cells (Figure 7F). Unexpectedly, however, despite the fact that SQSTM1 mRNA levels were not increased, SQSTM1/ p62 protein levels were higher in LP-1/Cfz cells (Figure $7 \mathrm{~F})$.

Kampmann and colleagues recently reported that knockdown of the $19 \mathrm{~S}$ proteasome subunit genes desensitized U266 MM cells to carfilzomib [65]. Because the $19 \mathrm{~S}$ regulator delivers substrates to the $20 \mathrm{~S}$ catalytic core, they hypothesized that a loss in 19S function may lead to the selective accumulation of certain proteins. SQSTM1/p62 was one of the proteins that accumulated upon knockdown [65]. Congruent with their results, GSEA indicated that many of the proteasome 19S subunit genes were downregulated in LP-1/Cfz cells (Figure $\mathrm{S} 7$ ). Therefore, it is possible that this mechanism may contribute to increased SQSTM1/p62 levels in LP-1/ $\mathrm{Cfz}$ cells. However, as described below, we found that elevated protein synthesis was a contributing factor to the observed differences in SQSTM1/p62 levels. In this context, it is worth mentioning that knockdown of the

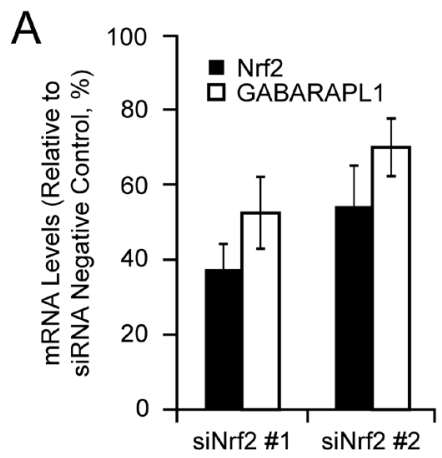

C
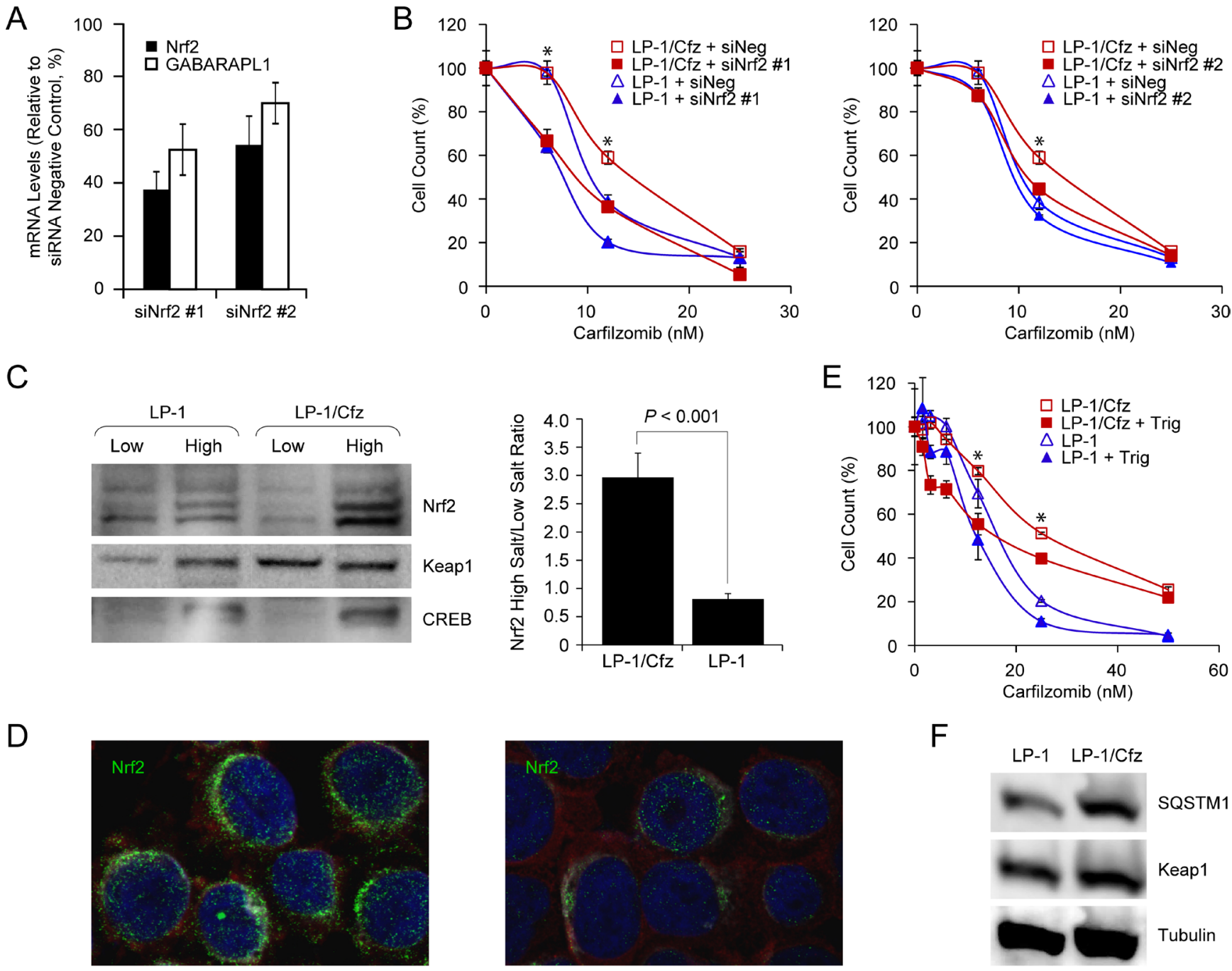

$\mathrm{LP}-1 / \mathrm{Cfz}$
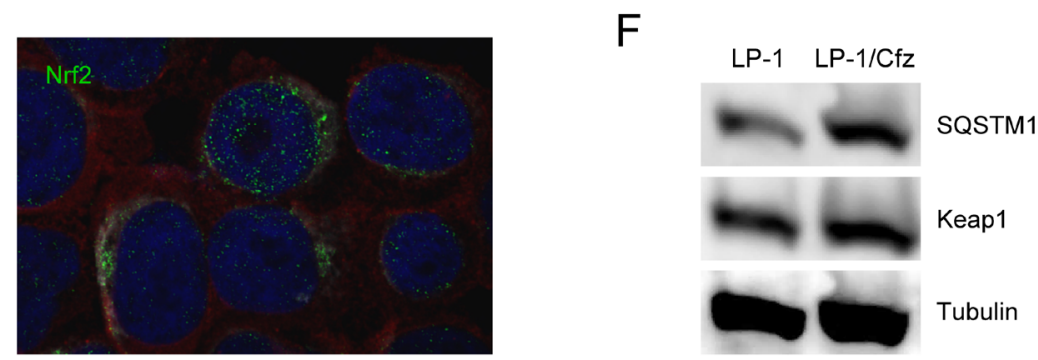

Figure 7: Nrf2 activation in LP-1/Cfz cells confers resistance to carfilzomib. A. Knockdown of Nrf2 mRNA using two specific siRNAs (siNrf2 \#1, siNrf2 \#2) was accompanied by downregulation of GABARAPL1 mRNA (mean values of three qRT-PCR experiments). B. Knockdown of Nrf2 mRNA using two specific siRNAs (siNrf2 \#1, siNrf2 \#2) sensitizes LP-1/Cfz cells to carfilzomib. Cells were treated with the indicated concentrations of carfilzomib for 48 hours after transient transfection and cell viability was determined by alamarBlue assay. ${ }^{*}, P<0.001$ vs negative siRNA control (siNeg, $n=3$ ). C. Western blot analysis demonstrating significantly higher Nrf2 levels in the high salt fraction of LP-1/Cfz cell lysates $(P<0.001 ; n=4)$. D. Cells were labeled with anti-Nrf2 antibody (Alexa Fluor 488 , green) and immunofluorescence staining was analyzed by confocal laser scanning microscopy. E. Cells were treated with the indicated concentrations of carfilzomib for 48 hours in the absence or presence of trigonelline (Trig, $1 \mu \mathrm{M}$ ), an inhibitor of Nrf 2 nuclear import, and cell viability was determined by alamarBlue assay. ${ }^{*}, P<0.001 v s$ carfilzomib alone $(n=3)$. F. Western blot analysis showing increased SQSTM1 levels in LP-1/Cfz compared to parental LP-1 cells $(P<0.03 ; n=5)$. 
19S proteasome subunit genes in U266 MM cells was not associated with substantially increased 20 S chymotrypsinlike protease activity selectively inhibited by carfilzomib [65]. Similarly, we did not detect a significant increase in 20S chymotrypsin-like protease activity in LP-1/Cfz cells (data not shown), which was consistent with only a slight $(\sim 1.3$-fold $)$ increase in mRNA of the PSMB5-encoded constitutive $\beta 5$ subunit targeted by carfilzomib (Figure S7).

Kampmann and colleagues also identified genes involved in other nodes of the proteostasis network that desensitized U266 MM cells to carfilzomib, including
A
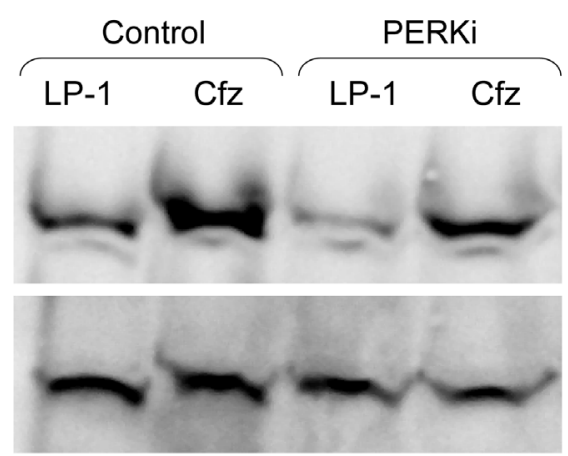

$\mathrm{B}$
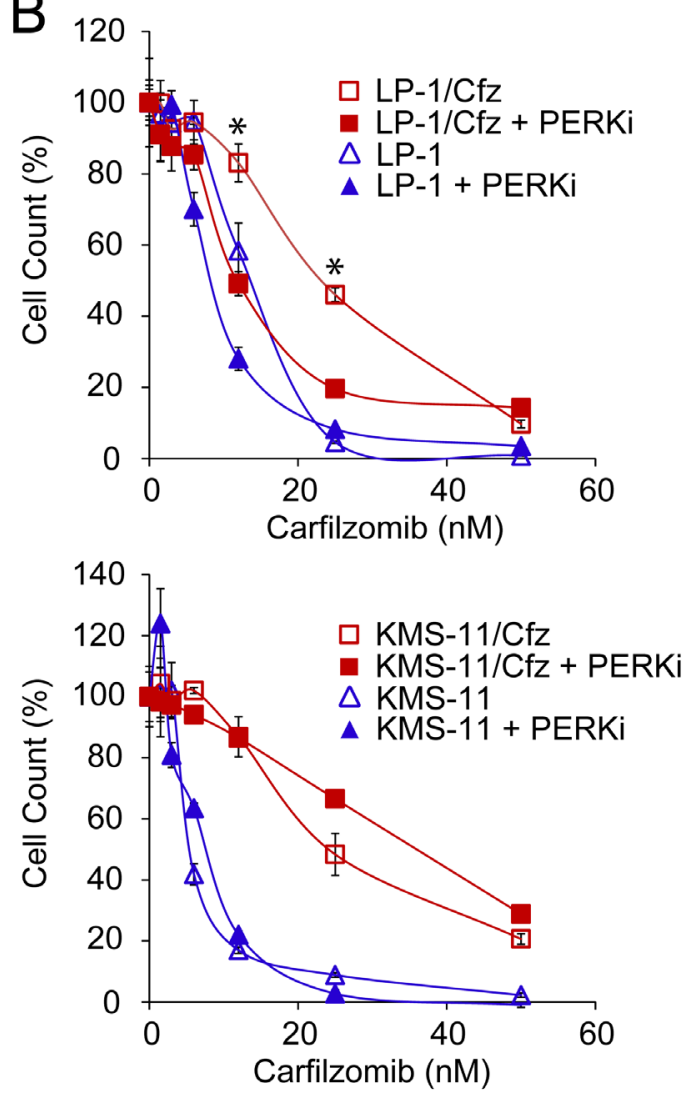

C
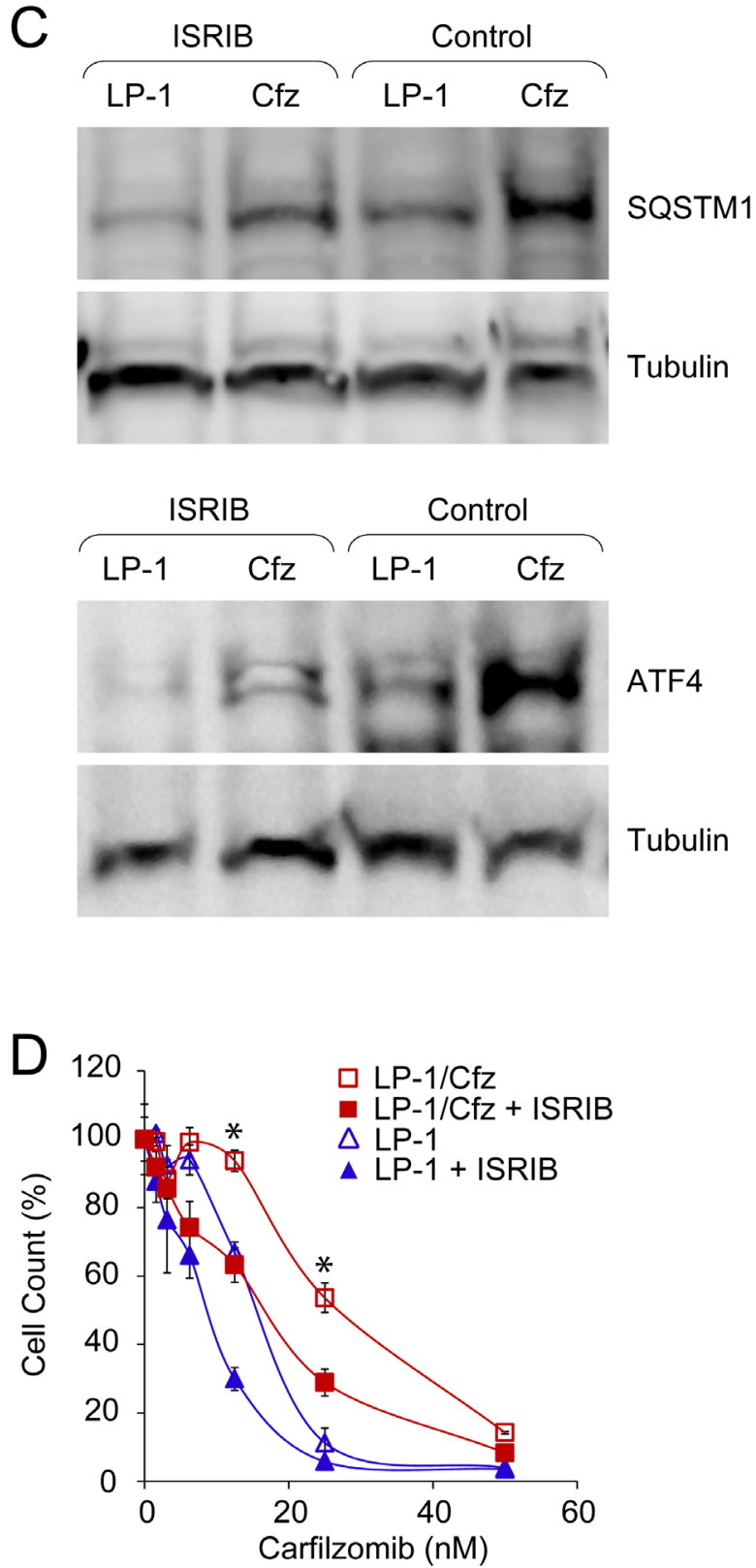

Figure 8: Constitutive PERK-eIF2 $\alpha$ signaling is associated with carfilzomib resistance in LP-1/Cfz cells. A. Western blot analysis showing that treatment with a PERK inhibitor (PERKi) lowers SQSTM1 levels in LP-1/Cfz (Cfz) and parental LP-1 cells $(P$ $<0.05 ; n=8)$. Cells were treated with MG-132 $(15 \mu \mathrm{M})$ for 18 hours in the absence or presence of GSK2656157 (10 $\mu \mathrm{M})$. B. Inhibition of PERK sensitizes LP-1/Cfz (top graph) but not KMS-11/Cfz cells (bottom graph) to carfilzomib. Cells were treated with the indicated concentrations of carfilzomib for 72 hours in the absence or presence of GSK2656157 (PERKi, $10 \mu \mathrm{M})$ and cell viability was determined by alamarBlue assay. *, $P<0.001 v s$ carfilzomib alone $(n=3)$. C. Western blot analysis showing that ISRIB treatment decreases SQSTM1 levels $(P<0.03 ; n=3)$ (top panels) and ATF4 levels (bottom panels) in LP-1/Cfz and parental LP-1 cells. Cells were treated with MG-132 $(15 \mu \mathrm{M})$ for 18 hours in the absence or presence of ISRIB $(20 \mathrm{nM})$. D. Inhibition of eIF2 $\alpha$ sensitizes LP-1/Cfz cells to carfilzomib. Cells were treated with the indicated concentrations of carfilzomib for 48 hours in the absence or presence of ISRIB (20 nM) and cell viability was determined by alamarBlue assay. ${ }^{*}, P<0.001 v s$ carfilzomib alone $(n=3)$. 
components of the eIF4F translation initiation complex (for example, EIF4E1) and the mechanistic target of rapamycin (MTOR) [65]. GSEA indicated that both of these pathways were downregulated in LP-1/Cfz cells: BIOCARTA_EIF4_PATHWAY $(P<0.001)$ (Figure S8A) and HĀLLMARK_MTORC1_SIGNALING $(P<$ 0.009) (Figure S8B). Since activation of the PERK-ATF4 pathway was also implicated by GSEA (Figure S3A) and PERK inhibits mTORC1 during induction of prosurvival autophagy [66], we assessed potential involvement of the PERK-eIF2 $\alpha$ axis on SQSTM1/p62 translation. For these studies, we tested a highly selective inhibitor of PERK kinase activity (GSK2656157) [67] or another small molecule (ISRIB) which acts downstream and reverses the effects of phosphorylated eIF $2 \alpha$, thereby blocking ATF4 synthesis [68]. We found that PERK inhibition significantly reduced SQSTM1/p62 levels in LP-1/Cfz cells (Figure 8A) and sensitized LP-1/Cfz cells to carfilzomib (Figure $8 \mathrm{~B}$, top graph). By comparison, KMS-11/Cfz cells were not sensitized by PERK inhibition (Figure 8B, bottom graph). Treatment of LP-1/Cfz cells with ISRIB also decreased SQSTM1/p62 levels (Figure $8 \mathrm{C}$, top panels) $(P<0.03 ; n=3)$ while concomitantly diminishing ATF4 levels (Figure 8C, bottom panels). Further, ISRIB treatment sensitized LP-1/Cfz cells to carfilzomib (Figure 8D). Thus, these results indicated that activation of the PERK-eIF2 $\alpha$ pathway contributed to carfilzomib resistance. Moreover, they argued that SQSTM1/p62 translation occurs in part via an ATF4-like uORF-mediated mechanism (see Table S2 of ref. [69] for details of SQSTM1 uORF-containing transcripts).

Interestingly, we did not observe enrichment of IRE1 or ATF6 expression signatures corresponding to the other branches of the UPR in LP-1/Cfz cells. In fact, GSEA indicated that UPR signaling was attenuated (HALLMARK_UNFOLDED_PROTEIN_RESPONSE downregulated; Figure S8C). Therefore, the mechanistic basis for PERK activation in LP-1/Cfz cells is unknown. However, alternative signals for PERK activation have recently been uncovered $[28,29,66,70]$. In particular, Gupta and colleagues described a noncanonical mechanism of PERK-Nrf2 activation in the absence of an endoplasmic reticulum stress response that results from an epithelial-to-mesenchymal transition (EMT) $[28,70]$. Along these lines, enrichment of an EMT-like expression signature (HALLMARK_EPITHELIAL_ MESENCHYMAL_TRANSITION) was indicated by GSEA in LP-1/Cfz cells (Figure S8D), and decreased cell surface expression of E-cadherin compared to parental LP-1 cells (Figure S8E) is consistent with this as a contributory mechanism $[71,72]$.

\section{Induction of $\mathrm{Nrf} 2$ target genes in $\mathrm{LP}-1 / \mathrm{Cfz}$ cells creates a positive feedback loop promoting eIF4E3-driven Nrf2 translation}

Although Nrf2 levels are primarily governed by interaction with Keap1, accumulating evidence has highlighted the importance of de novo translation [22-24]. Therefore, we were prompted to compare Nrf2 synthesis in LP-1/Cfz and parental LP-1 cells. For these analyses, the cells were pretreated with MG-132 to prevent Nrf2 proteasomal degradation. Under these conditions, we found significantly higher levels of Nrf2 in LP-1/Cfz cells (Figure 9A). We next tested the effects of 4EGI-1, an inhibitor of cap-dependent translation that prevents binding of eukaryotic initiation factor $4 \mathrm{G}$ (eIF4G) to eIF4E family members (the best characterized of which is eIF4E1) within the eIF4F translation initiation complex [73]. Inhibition of the eIF4E/eIF4G interaction eliminated the differences in Nrf2 levels between LP-1/Cfz and parental LP-1 cells (Figure 9B). The data thus indicated more active cap-dependent synthesis of Nrf2 in LP-1/Cfz cells. This result was contrary to the GSEA prediction that canonical eIF4F-mediated translation initiation was downregulated in LP-1/Cfz compared to parental LP-1 cells (Figure S8A) and suggested the possible involvement of an alternative eIF4F cap-binding complex containing another member of the eIF4E family [74]. Along these lines, it has recently been appreciated that eIF4E3 interacts with eIF4G and competes with eIF4E1 to form a novel eIF4F cap-binding complex $[37,38]$. Because this $\mathrm{Nrf} 2$ target was upregulated at the mRNA level in LP-1/Cfz cells (Table S1A), we were interested in whether this was reflected at the level of eIF4E3 protein. We found that the increased EIF4E3 mRNA levels in LP-1/Cfz cells were accompanied by elevated eIF4E3 protein levels, whereas LP-1/Cfz and parental LP-1 cells had similar levels of eIF4E1 (Figure 9C).

Phosphorylation of eIF4E1 by mitogen-activated protein kinase interacting kinases (MNK) 1 and 2 stimulates translation of a subset of mRNAs encoding proteins involved in cancer development and progression [75]. Gartenhaus and colleagues found that treatment of diffuse large B-cell lymphoma cells with an MNK inhibitor, CGP57380, reduced eIF4E1-driven translation and resulted in a compensatory increase in eIF4E3-driven translation [38]. To determine whether Nrf2 translation might be regulated via eIF4E1-eIF4E3 interplay, we investigated the effect of MNK inhibition on Nrf2 protein levels in LP-1/Cfz and parental LP-1 cells. We found that after CGP57380 treatment Nrf2 levels increased in both cases, with the levels in parental LP-1 cells approaching those in LP-1/Cfz cells (Figure 9B). These findings supported a role of eIF4E3 in the elevated synthesis of Nrf2 and the carfilzomib-resistant phenotype of LP-1/ Cfz cells. To directly test this, we transfected LP-1/Cfz 
A

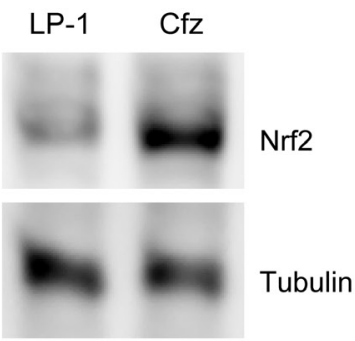

C
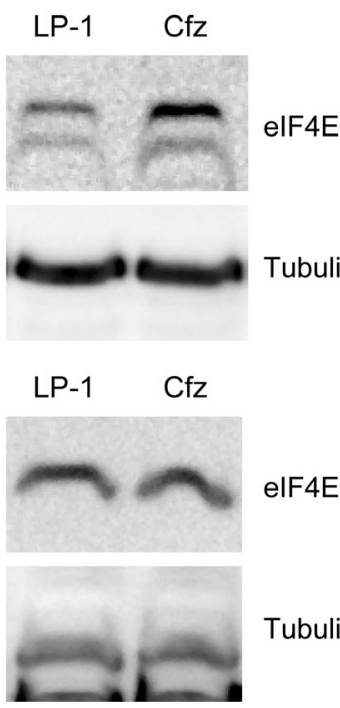

elF4E1
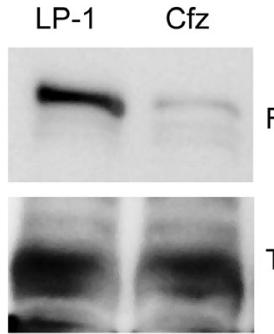

FAM129A
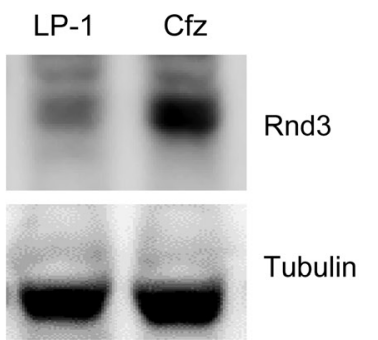

$\mathrm{B}$
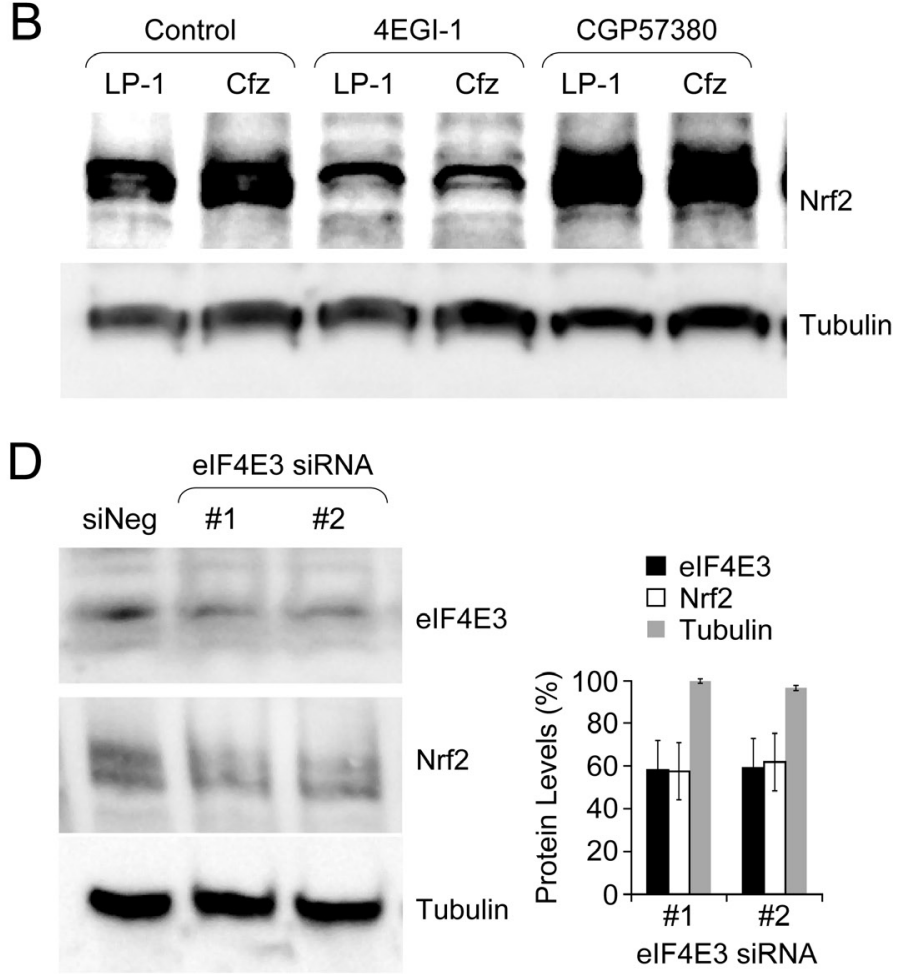

Figure 9: Acquisition of carfilzomib resistance in $\mathrm{LP}-1 / \mathrm{Cfz}$ cells is associated with eIF4E3-mediated translational reprogramming. A. Western blot analysis showing increased Nrf2 levels in LP-1/Cfz (Cfz) versus parental LP-1 cells $(P<0.002 ; n=$ 18). Cells were treated with MG-132 $(15 \mu \mathrm{M})$ for 18 hours. B. Synthesis of Nrf2 depends on eIF4E/eIF4G interaction $(P<0.003 ; n=10)$ and is enhanced upon MNK inhibition $(P<0.03 ; n=6)$. Cells were treated with MG-132 $(15 \mu \mathrm{M})$ for 18 hours in the absence or presence of an eIF4E/eIF4G interaction inhibitor (4EGI-1, 50 $\mu \mathrm{M}$ ) or an MNK inhibitor (CGP57380, $10 \mu \mathrm{M})$ and whole cell lysates were analyzed by Western blotting. C. Western blot analysis showing (from top to bottom) increased eIF4E3 levels $(P<0.001 ; n=4)$, similar eIF4E1 levels, decreased FAM129A levels $\left(P<1 \times 10^{-4} ; n=7\right)$, and increased Rnd3 levels $(P<0.02 ; n=6)$ in LP-1/Cfz versus parental LP-1 cells. Cells were treated with MG-132 $(15 \mu \mathrm{M})$ for 18 hours. D. Western blot analysis showing that knockdown of EIF4E3 mRNA using two specific siRNAs was accompanied by decreased eIF4E3 and Nrf2 protein levels. E. Knockdown of EIF4E3 mRNA (siRNA \#1) sensitizes LP-1/ Cfz cells to carfilzomib. Cells were treated with the indicated concentrations of carfilzomib for 48 hours after transient transfection and cell viability was determined by alamarBlue assay. ${ }^{*}, P<0.001 v s$ negative siRNA control (siNeg, $n=3$ ). 
and parental LP-1 cells with two specific EIF4E3 siRNAs and a negative control siRNA. Figure 9D shows that Nrf2 protein levels decreased with eIF4E3 protein knockdown. Moreover, eIF4E3 knockdown sensitized LP-1/Cfz cells to carfilzomib (Figure 9E).

We also examined whether the protein levels of two other novel Nrf2 targets involved in the control of cap-dependent translation initiation - RND3/RhoE and FAM129A/Niban - corresponded with the differential mRNA levels observed in LP-1/Cfz and LP-1 cells (Figure S4). FAM129A inhibits PERK-mediated phosphorylation of eIF $2 \alpha$ [50]. Additionally, FAM129A and Rnd3 reciprocally modulate the activity of $4 \mathrm{E}-\mathrm{BP} 1$, an inhibitor of eIF4E1 $[49,50]$. Briefly, the dephosphorylated form of 4E-BP1 binds to eIF4E1, preventing binding of eIF4G and formation of the eIF4F complex. mTORC1 phosphorylates 4E-BP1, leading to its dissociation from eIF4E1 which allows eIF4F formation and translation initiation [75]. FAM129A positively affects mTORC1 phosphorylation of 4E-BP1, thereby facilitating eIF4E1-driven translation [50]. In contrast, Rnd3 inhibits mTORC1 phosphorylation of 4E-BP1 which prevents 4E-BP1 disassociation from eIF4E1 and therefore inhibits eIF4E1 function [49]. This mechanism only pertains to eIF4E1 since 4E-BP1 does not bind to eIF4E3 [74]. FAM129A protein levels were decreased in LP-1/Cfz cells corresponding with its diminished mRNA expression. Conversely, Rnd3 protein levels were increased in LP-1/Cfz cells concomitant with increased mRNA expression (Figure 9C). Collectively, these results are consistent with a potential positive feedback process associated with acquisition of carfilzomib resistance in LP-1/Cfz cells wherein initial activation of Nrf2 leads to direct and indirect regulation of target genes - including transcriptional upregulation of EIF4E3 - that facilitate enhanced eIF4E3-driven translation of Nrf2.

\section{Increased $E I F 4 E 3$ expression predicts Nrf 2 target gene activation in minimal residual disease and relapsed MM patient samples}

To assess the clinical relevance of our findings, we analyzed three independent publicly available gene expression datasets of MM patients. We first examined the gene expression profiles of chemoresistant minimal residual disease (MRD) and matched diagnostic samples from MM patients included in the GEM2010MAS65 clinical trial (GEO accession number GSE70399) [76]. Notably, EIF4E3 expression was found to be increased in 5 out of 7 MRD samples ( $\mathrm{FC}=1.25 ; P=0.013)$. When GSEA was applied to these 5 cases, significant upregulation of Nrf2 target genes (NFE2L2.V2 gene set) was observed in the persisting MRD cells (Figure 10A). As was found for LP-1/Cfz cells, UPR signaling was attenuated (HALLMARK_UNFOLDED_PROTEIN
RESPONSE downregulated; Figure 10B). Moreover, GSEA predicted an inverse relationship with an enrichment pattern corresponding to genes regulated by EIF4E1 overexpression (Figure 10C).

We also examined patient-paired relapse and diagnostic samples from $17 \mathrm{MM}$ patients treated with various regimens (GEO accession number GSE36824) [77]. EIF4E3 expression was increased in 4 out of 17 cases of disease progression. GSEA indicated that $\mathrm{Nrf} 2$ target genes (NFE2L2.V2 gene set) were upregulated during disease course in these 4 cases (Figure 11A). As for MRD cells with increased EIF4E3 expression, GSEA predicted lack of UPR stress in the relapsed MM cells (HALLMARK_UNFOLDED_PROTEIN_RESPONSE downregulated; Figure 11B). These relapsed MM cells also had enrichment of an EMT-like expression signature (HALLMARK_EPITHELIAL_MESENCHYMAL_ TRANSITION; Figure 11C). Moreover, genes upregulated through activation of the mTORC1 complex were downregulated in these 4 cases (HALLMARK MTORC1_SIGNALING; Figure 11D). In addition, GSEA again predicted an inverse correlation with an EIF4E1specific gene expression signature (Figure 11E). Of the 17 samples, GABARAPL1 expression was increased in 5 cases during disease progression, two of which also had increased EIF4E3 expression. Performing GSEA on the combined EIF4E3 plus GABARAPL1 dataset (7 out of 17 cases) indicated activation of Nrf2 signaling and yielded the same enrichment patterns for the other pathways identified for the 4 cases with increased EIF4E3 expression (data not shown).

On the other hand, when GSEA was performed on the 10 relapsed MM patient samples that did not exhibit increased EIF4E3 or GABARAPL1 expression, the exact opposite patterns of enrichment were obtained. In these cases, a positive correlation with EIF4E1-upregulated genes was observed during disease progression (Figure $11 \mathrm{~F}$ ) concomitant with a prediction of increased mTORC1 signaling (Figure S9A). Although a UPR was predicted (Figure S9B), Nrf2 target genes were downregulated (Figure S9C) and a reverse EMT-like expression signature (i.e., mesenchymal-to-epithelial transition) was suggested (Figure S9D). Therefore, these parameters appeared to separate the relapsed MM samples into two distinct groups: one, "LP-1/Cfz-like", characterized by increased EIF4E3 (and/or GABARAPL1) expression having activation of the Nrf2 signaling pathway, and a second group ("eIF4E1-like") exhibiting the opposite phenotype and pathway activation states in which enhanced eIF4E1driven translation was implied. 

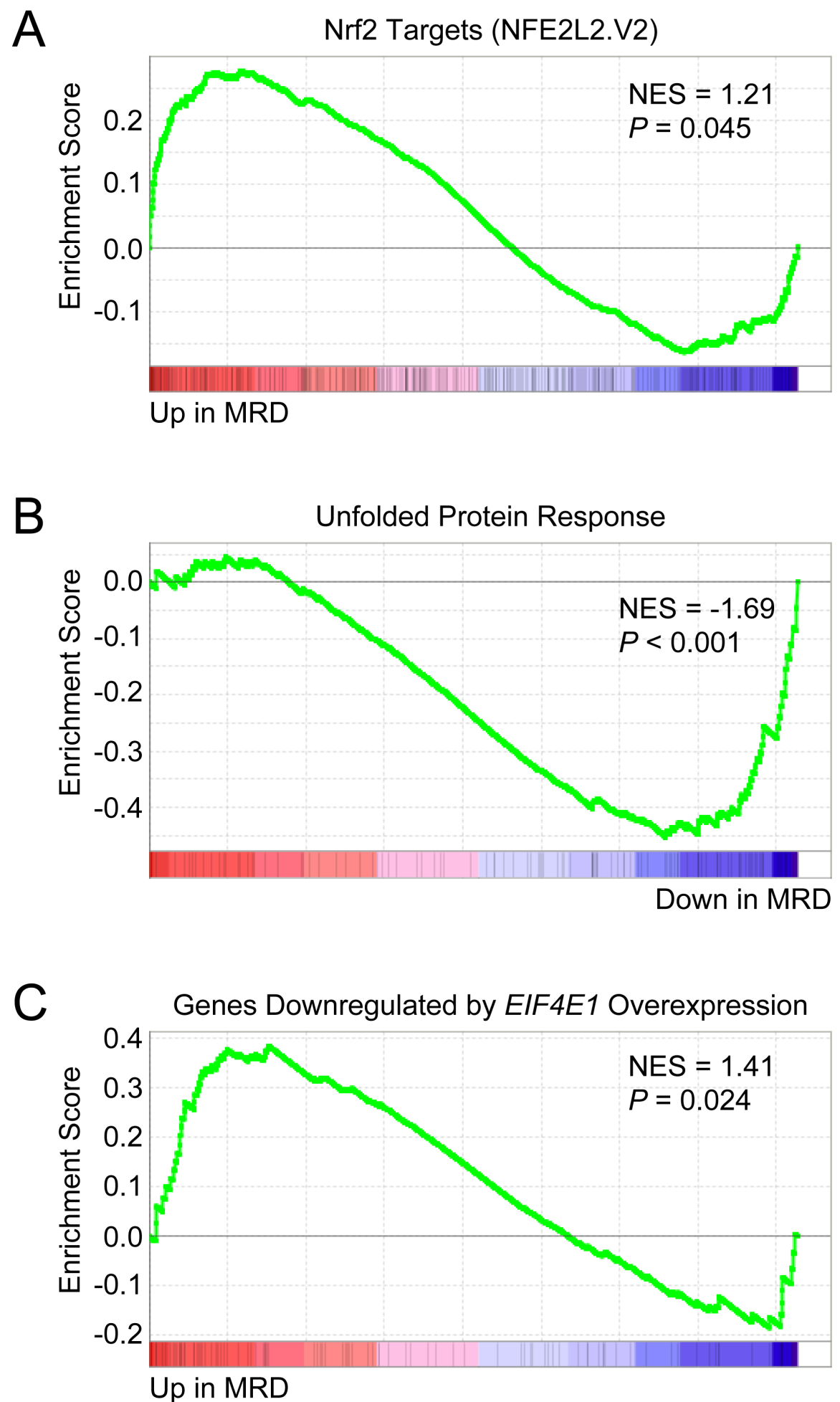

Figure 10: Chemoresistant minimal residual disease (MRD) cells from MM patients have increased EIF4E3 expression and Nrf2 target gene signature enrichment. Microarray data was obtained from the Gene Expression Omnibus database (www. ncbi.nlm.nih.gov/geo/; GEO accession number GSE70399) for matched MRD and diagnostic plasma cells from MM patients enrolled in the GEM2010MAS65 trial (ClinicalTrials.gov Identifier: NCT01237249). EIF4E3 expression in MRD cells versus baseline was analyzed with the GEO2R web tool (http://www.ncbi.nlm.nih.gov/geo/geo2r/) and was found to be increased in 5 out of 7 samples (average fold change $=1.25 ; P=0.013$ ). A. GSEA indicated that Nrf2 target genes were upregulated in MRD cells. Gene set: NFE2L2.V2 (M2870). B. GSEA indicated that genes upregulated during the unfolded protein response were downregulated in MRD cells. Gene set: HALLMARK UNFOLDED_PROTEIN_RESPONSE (M5922). C. GSEA indicated that genes downregulated in primary human mammary epithelial cells upon overexpression of EIF4E1 were upregulated in MRD cells. Gene set: EIF4E_DN (M2790). 


\section{Elevated expression of EIF4E3 is prognostic of poor survival}

LP-1 MM cells contain the $t(4 ; 14)$ chromosomal translocation that activates a histone methyltransferase encoded by the WHSCl gene (also known as MMSET)

A

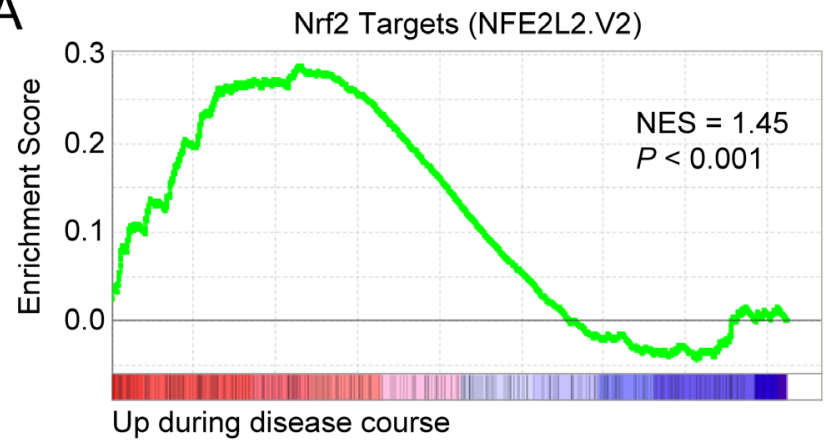

C

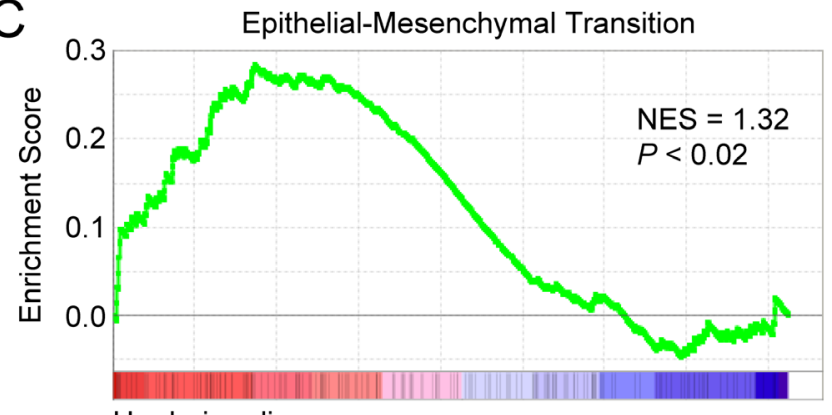

Up during disease course

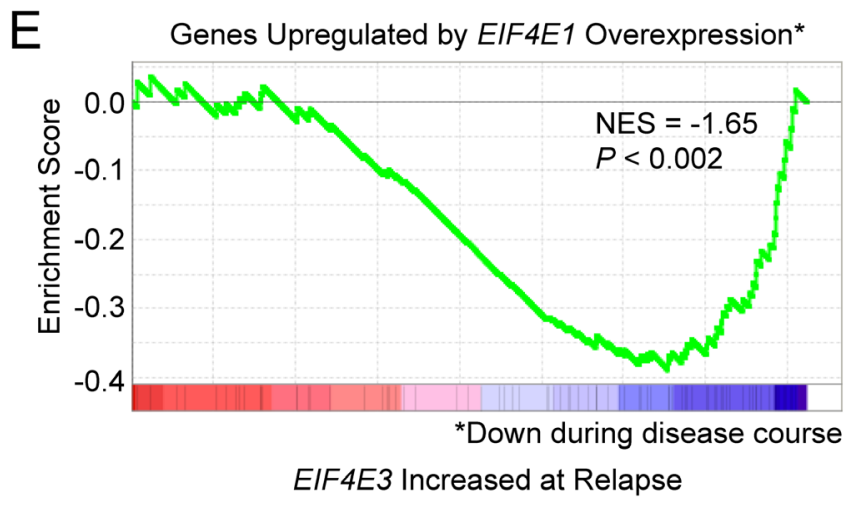

[78], which is consistently associated with poor outcome [79]. Using the UAMS-70 prognostic gene signature, Shaughnessy and colleagues separated WHSCl-positive MM patients into higher-risk and lower-risk subgroups [80]. We were curious to know whether increased expression of EIF4E3 was prognostic for decreased overall survival of WHSC1-positive MM patients. Using

B

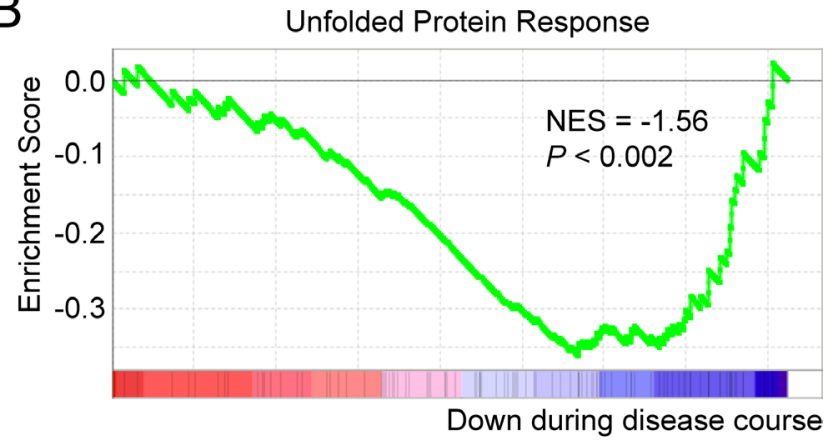

$\mathrm{D}$

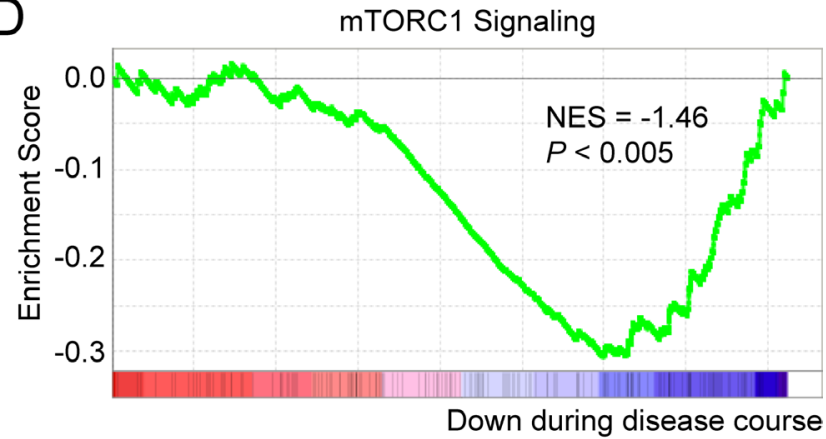

F

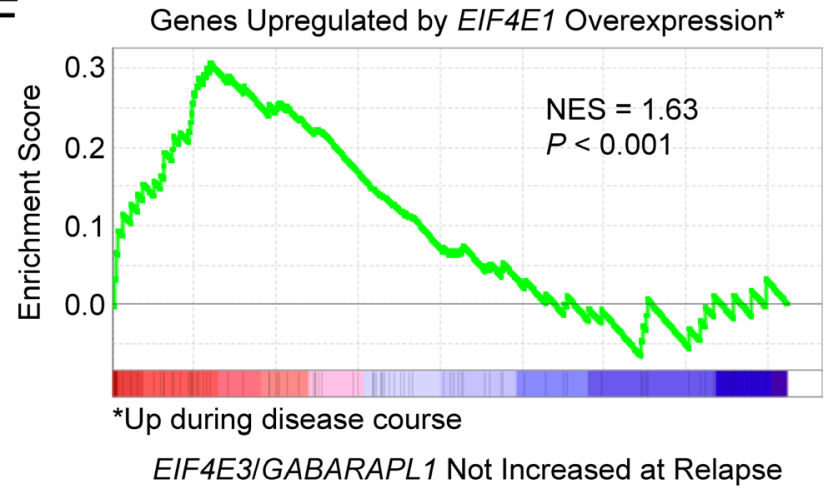

Figure 11: Gene expression signatures of MM cells associated with increased EIF4E3 expression during progression of disease. Microarray data was obtained from the GEO database (GEO accession number GSE36824) for patient-paired relapse and diagnostic samples from 17 patients treated with various regimens. EIF4E3 expression was increased in 4 out of 17 cases. A. GSEA indicated that Nrf2 target genes were upregulated during disease course in these cases. Gene set: NFE2L2.V2 (M2870). B. GSEA indicated that genes upregulated during the UPR were downregulated. Gene set: HALLMARK_UNFOLDED_PROTEIN_RESPONSE (M5922). C. These patients had enrichment of an EMT-like expression signature. Gene set: HALLMARK_EPITHELIAL_MESENCHYMAL TRANSITION (M5930). D. Genes upregulated through activation of the mTORC1 complex were also downregulated in these cases. Gene set: HALLMARK_MTORC1_SIGNALING (M5924). E. GSEA indicated that genes upregulated in primary human mammary epithelial cells upon overexpression of $E I F 4 E 1$ were downregulated during disease course in relapse cells with increased EIF4E3 expression. Gene set: EIF4E_UP (M2791). F. GSEA of patient-paired relapse and diagnostic samples without increased EIF4E3 and/or GABARAPL1 expression (10 out of 17 cases) showed enrichment of the EIF4E_UP signature. See also Figure S9. 
the PROGgeneV2 prognostic biomarker identification tool [81] as previously described [11], we performed a Cox proportional hazards regression analysis on 546 newly diagnosed MM patients treated on the UARK 98026 TT2 and UARK 2003-033 TT3 clinical trials [80]. The hazard ratio (HR) for patients with high versus low WHSC1 expression was 1.3 (95\% confidence interval (CI), 1.1-1.54; $P<0.002$ ). As shown in Figure 12A, highlevel coexpression of EIF4E3 resulted in increased risk of mortality $(\mathrm{HR}=1.6 ; 95 \% \mathrm{CI}, 1.17-2.21 ; P<0.004)$, which was higher than the increased risk score predicted by high-level coexpression of EIF $4 E 1$ ( $\mathrm{HR}=1.45 ; 95 \%$ CI, 1.04-2.02; $P<0.03$ ) (Figure 12B).

\section{DISCUSSION}

Despite improved outcomes with the introduction of novel agents such as proteasome inhibitors to MM treatment regimens over the past decade, patients inevitably succumb to their disease because the MM cells become resistant to the drugs [1]. MM cells can develop resistance to proteasome inhibitors through a variety of mechanisms [4]. However, it is still unclear which mechanisms explain clinical proteasome inhibitor resistance.

In this paper we report the establishment of a new carfilzomib-resistant MM cell line derivative, LP-1/Cfz, in which elevated levels of the SQSTM1/p62 ubiquitinbinding cargo receptor were associated with carfilzomib resistance that comprised both prosurvival autophagy and Nrf2 pathway activation. Our results support a model wherein activation of the SQSTM1/p62-Nrf2 pathway — in concert with the PERK-eIF2 $\alpha /$ ATF4 axis — directs reprogramming of MM cells, modulating redox and energy homeostasis via elevated FAO and inducing prosurvival autophagy involving GABARAPL1 upregulation [15, 2629, 34-36]. We suggest that the increased SQSTM1/p62 levels allow newly synthesized Nrf2 to escape Keap1mediated sequestering in the cytoplasm [13, 14], resulting in the regulation of a subset of its target genes, including EIF4E3, which in turn facilitate enhanced translation of Nrf2 and establish a positive feedback loop. While further mechanistic studies are required to fully define the role played by SQSTM1/p62 in carfilzomib resistance, we demonstrated the direct involvement of Nrf2 by genetic and pharmacologic inhibition and a contribution of PERK using two highly selective small-molecule inhibitors of the PERK-eIF2 $\alpha$ axis $[67,68]$.

Previously we reported that acquisition of carfilzomib resistance in KMS-11/Cfz and KMS-34/Cfz MM cells was associated with prosurvival autophagy involving SQSTM1/p62 [11]. Comparative analyses of KMS-11/Cfz cells herein also revealed activation of Nrf2 target genes (Figure 1B). Carfilzomib resistance in KMS-11/Cfz cells is associated with KLF4-mediated transcriptional upregulation of SQSTM1 expression [11]. Others have described the establishment of a positive feedback loop in which Nrf2 transcriptionally activates SQSTM1 [13, 14] (highlighted in Figure S1B). We note that KLF4 is also an Nrf2 target gene (Table S1B), so we envision a model in KMS-11/Cfz cells wherein KLF4 and Nrf2 cooperate to transcriptionally maintain elevated SQSTM1/p62 levels.

GSEA revealed that activation of the PERK-eIF $2 \alpha$ axis in LP-1/Cfz cells was not associated with induction of a UPR (Figure S8C). Gupta and colleagues have described
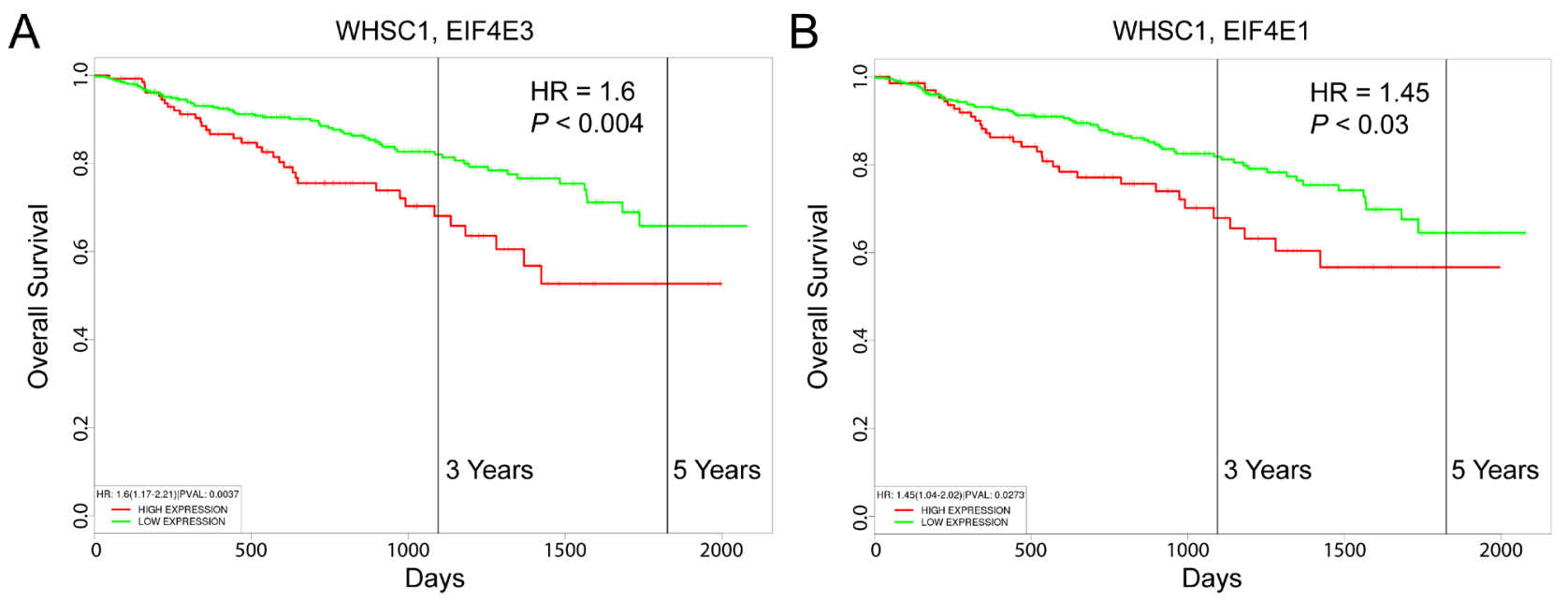

Figure 12: Prognostic value of EIF4E3 expression in MM patient survival outcomes. Kaplan-Meier survival plots of 546 newly diagnosed patients with MM consisting of 351 cases enrolled on total therapy 2 (TT2) and 195 patients enrolled in total therapy 3 (TT3) which incorporates bortezomib into the induction phase of the TT2 transplant regimen (GEO accession number GSE2658) were created using PROGgeneV2. A. Coexpression of WHSC1 and EIF4E3. B. Coexpression of WHSC1 and EIF4E1. Cohorts were divided at the $75^{\text {th }}$ percentile of mean gene expression. HR, hazard ratio determined by Cox proportional hazards model. 
a noncanonical mechanism of PERK-Nrf2 activation in the absence of an endoplasmic reticulum stress response that involves an EMT-like adaptation [28, 70]. Enrichment of an EMT-like expression signature was predicted by GSEA for LP-1/Cfz cells (Figure S8D) and for relapsed MM patient samples with increased EIF4E3 expression (Figure 11C). Further investigation of an EMT-related mechanism is underway and our preliminary studies are suggestive of crosstalk among Nrf2, ERBB2 and hypoxia signaling pathways [82, 83]. Indeed, our ongoing examination of LP-1/Cfz cells indicates that they exhibit intermediate EMT-like characteristics [84]. Of relevance in this regard, a recent study by Orlowski and colleagues identified low levels of TJP1 encoding the epithelial marker tight junction protein 1 (also known as zonula occludens 1 ) as a determinant of myeloma proteasome inhibitor resistance [85]. During EMT, intercellular junctions are disrupted and TJP1 is coordinately downregulated with E-cadherin [86]. Interestingly, TJP1 expression was decreased in $\mathrm{KMS}-11 / \mathrm{Cfz}$ and KMS-34/Cfz cells but increased in LP-1/Cfz cells (our unpublished results). While Nrf2 has been demonstrated to downregulate E-cadherin expression [72], it has been implicated in the upregulation of TJP1 expression [87]. Intriguingly, Orlowski and colleagues reported that proteasome inhibitor sensitivity associated with increased TJP1 expression was due to suppression of expression of the PSMB 8 gene encoding the immunoproteasome $\beta 5 \mathrm{i} / \mathrm{LMP} 7$ (chymotrypsin-like) subunit targeted by carfilzomib and bortezomib as well as the $P S M B 9$ gene encoding the $\beta 1 \mathrm{i} / \mathrm{LMP} 2$ (caspase-like) subunit that is also targeted by bortezomib [85]. In accord with their findings of an inverse relationship with TJP1 expression, both $P S M B 8$ and $P S M B 9$ were downregulated in LP-1/Cfz cells (Figure S7). In their model, this apparently occurred through TJP1-mediated suppression of EGFR/ERBB2 signaling [85]. As TJP1 also interacts with GABARAPL1 through the CUL3-KBTBD6/ KBTBD7 ubiquitin ligase in the autophagy network [53], we speculate that complex interplay between Nrf2GABARAPL1/TJP1 and ERBB2 signaling pathways may underlie the carfilzomib-resistant LP-1/Cfz phenotype (Figure S3C, S3D) [45, 46, 88, 89].

Carfilzomib resistance in the KMS-11/Cfz and KMS-34/Cfz models was characterized by a partial reversal of plasma cell maturation [11]. These results are in line with the findings of others showing that primary MM cells are capable of dedifferentiating into a less mature MM phenotype conferring experimental and clinical drug resistance [90-92]. It is notable that LP-1/Cfz cells did not fully recapitulate this pattern. Parental LP-1 cells exhibit a membrane phenotype that is intermediate between late B lymphocytes and plasma cells based on MHC class II antigen expression [39]. Like the KMS-11/ Cfz and KMS-34/Cfz carfilzomib-resistant MM models [11], acquisition of carfilzomib resistance in LP-1/ Cfz cells was accompanied by decreased expression of
SLAMF7 encoding the plasma cell-specific CD319 cell surface marker, reflecting a partial reversal of plasma cell maturation (Table S1A). Conversely, MHC class II antigen expression was diminished, which is reminiscent of plasmacytic differentiation (Table S2A). In this latter respect, LP-1/Cfz cells might resemble in part the situation observed in mantle cell lymphoma where bortezomib resistance increased with plasmacytic differentiation [93]. Accordingly, LP-1/Cfz cells may have attained the "sweet spot" maturation stage intrinsically less susceptible to proteasome inhibition proposed by Tiedemann and colleagues [92].

Our results indicating a role of $\mathrm{Nrf} 2$ activation in experimental carfilzomib resistance parallel those of others wherein Nrf2 target gene activation was associated with poor responsiveness to bortezomib in mouse and human MM models [94, 95]. In this regard, both LP-1/ $\mathrm{Cfz}$ and $\mathrm{KMS}-11 / \mathrm{Cfz}$ exhibit some cross-resistance to bortezomib (Figure S1). In that various regimens were used in the treatment of the MM patients that we analyzed $[76,77,80]$, we cannot conclude that increased EIF4E3 expression can be used as a specific indicator of clinical carfilzomib resistance. Nonetheless, the finding that EIF4E3 expression is increased in certain chemoresistant minimal residual disease and relapsed MM patient samples and is predictive of $\mathrm{Nrf} 2$ target gene activation, strongly suggests that the Nrf2-EIF4E3 axis and eIF4E3driven translation contributes to $\mathrm{MM}$ drug resistance mechanisms in the clinical setting. On the other hand, lack of EIF $4 E 3$ expression in relapsed samples was able to distinguish a second group of MM patient samples exhibiting the opposite phenotype and pathway activation states in which enhanced eIF4E1-driven translation was indicated. These findings are consistent with the results of other studies demonstrating a role of eIF4E1 in MM biology and proteasome inhibitor resistance [65, 96-98]. It is particularly noteworthy therefore - especially in view of the documented oncogenic activity of EIF4E1 [75] that WHSC1-expressing MM patients with high EIF4E3 expression had less favorable outcomes than those with high EIF4E1 expression (Figure 12).

In summary, our finding of noncanonical SQSTM1/ p62-Nrf2 pathway activation adds to the growing appreciation from our work and others that carfilzomib resistance in MM can arise via multiple mechanisms $[10,11,65,85,99]$. MM is heterogeneous in its etiology and progression so these varied results are not entirely unexpected [100]. Collectively, our studies suggest several approaches to sensitize drug-resistant MM cells to carfilzomib $[10,11]$. In particular, the data presented herein support the development of novel therapies targeting the SQSTM1/p62-Nrf2 pathway for a subgroup of advanced stage MM patients with eIF4E3-driven translation. 


\section{MATERIALS AND METHODS}

\section{Cell culture}

LP-1 and KMS-11 MM cells were a kind gift from Dr. P. Leif Bergsagel (Mayo Clinic, Scottsdale, AZ) [101]. Cells were cultured in RPMI 1640 with GlutaMAX (Thermo Fisher Scientific) supplemented with 10\% fetal bovine serum (Cambrex BioScience), $100 \mathrm{U} / \mathrm{ml}$ penicillin and $100 \mu \mathrm{g} / \mathrm{ml}$ streptomycin. Cultures were maintained at $37^{\circ} \mathrm{C}$ in a humidified atmosphere containing $5 \% \mathrm{CO}_{2}$.

\section{Antibodies and reagents}

The following antibodies were used: anti-ATF4/ CREB-2 (c-20) (Santa Cruz Biotechnology, Cat. No. sc200); anti-eIF4E1 (P-2) (Santa Cruz Biotechnology, Cat. No. sc-9976); anti-eIF4E3 (Proteintech, Cat. No. 17282-1AP); anti-FAM129A/Niban (Signalway Antibody, Cat. No. 21401-2); anti-Keap1 (H-190) (Santa Cruz Biotechnology, Cat. No. sc-33569); anti-Nrf2 (H-300) (Santa Cruz Biotechnology, Cat. No. sc-13032); anti-RhoE/Rnd3 clone 4 (EMD Millipore Corporation, Cat. No 05-723); antiSQSTM1/p62 (Clone 3) mouse mAb (BD Transduction Laboratories, Cat. No. 610832); and anti- $\alpha$-tubulin mouse mAb (DM1A) (EMD Millipore Corporation, Cat. No. CP06). Carfilzomib (Cat. No. A-1098) was obtained from Active Biochem; chloroquine (Cat. No. S4157) and GSK2656157 (Cat. No. S7033) were purchased from Selleck Chemicals; (S)-4-carboxyphenylglycine (Cat. No. 0323), CGP57380 (Cat. No. 2731), 4EGI-1 (Cat. No. 4800) and MG-132 (Cat. No. 1748) were ordered from Tocris Bioscience; etomoxir (Cat. No. 11969) was obtained from Cayman Chemical; and trigonelline hydrochloride (Cat. No. T5509-1G) was from SigmaAldrich.

\section{Microarray gene expression analysis and quantitative real-time qRT-PCR validation}

Total RNA was isolated with the miRNeasy mini kit (Qiagen, Cat. No. 217004). Microarray gene expression analysis of triplicate samples was carried out by Expression Analysis, Inc. (Durham, NC) using Affymetrix GeneChip Human Genome U133 Plus 2.0 arrays. The data have been deposited in GEO (http:// www.ncbi.nlm.nih.gov/geo/) under accession number GSE78069. Reverse transcription was performed with the SuperScript VILO cDNA synthesis kit (Thermo Fisher Scientific, Cat. No. 11754250). Real-time qRTPCR was performed using the Power SYBR Green PCR master mix (Thermo Fisher Scientific, Cat. No. 4367659) on an ABI Prism 7000 Sequence Detection
System (Applied Biosystems) as previously described $[10,11]$. Primers synthesized by Sigma-Aldrich included: EEF1A2, forward, GTGTACAAGATTGGCGGCAT, reverse, GATGTTCACTGGCGCAAAGG; FAM129A, forward, TACATCCGAGGGAAAACTGAGG, reverse, GCCACAGAGTACTGACGACT; GABARAPL1, forward, AGGAGGACCATCCCTTTGAGT, reverse, TCTACAATCACGGGGACCCT; NFE2L2, forward, CGGTATGCAACAGGACATTG, reverse, TGGCTTCTGGACTTGGAACC; RND3, forward, GTCGGCTGCAAGTCTGATCT, reverse, CCATATTTGCCCCCTGGTCA; and ACTB, forward, GGACTTCGAGCAAGAGATGG, reverse, AGCACTGTGTTGGCGTACAG.

\section{Chromatin immunoprecipitation (ChIP)}

ChIP was performed on the GABARAPL1 promoter region with $20 \mu \mathrm{g}$ total chromatin and $5 \mu \mathrm{g}$ of anti-Nrf2 antibody using the SimpleChIP Enzymatic Chromatin IP Kit (Magnetic Beads) (Cell Signaling, Cat. No. 9003) as previously described [11], and $4 \%$ of the precipitated material was used per qPCR reaction. Background ChIP levels were obtained using $5 \mu \mathrm{g}$ of anti-Keap 1 antibody. Primers used were: GABARAPL1 promoter region, forward, CCGTGTCCTTCATCTGACTCC, reverse, TCGCTCCTGAACAGCAACAT.

\section{siRNA transfection}

For RNA interference, LP-1/Cfz and parental LP-1 cells were transiently transfected with Nrf2 siRNAs (SI03246950 [\#1] and SI03246614 [\#2]; QIAGEN, FlexiTube GeneSolution for NFE2L2, Product No. 1027416, Cat. No. GS4780), eIF4E3 siRNAs (s50217 [\#1] and s50218 [\#2]; Thermo Fisher Scientific Silencer Select) or a negative control siRNA (QIAGEN, ALLStars negative control siRNA, Cat. No. 1027281) using the HiPerFect transfection reagent (QIAGEN). Briefly, $2 \mathrm{x}$ $10^{6}$ cells per $\mathrm{ml}$ were seeded into 24 well plates in $100 \mu \mathrm{l}$ aliquots. Each well received a mixture of siRNAs (750 ng) and HiPerFect reagent (6 $\mu \mathrm{l})$ in $100 \mu \mathrm{l}$ serum-free culture medium preincubated for 15 minutes. After 5 hours, the cells were diluted to $6 \times 10^{5}$ per $\mathrm{ml}$ in complete medium. Cells $\left(3 \times 10^{5}\right.$ per $\left.\mathrm{ml}\right)$ were seeded into 96 well plates and treated with a range of carfilzomib concentrations. After 48 hours, cell growth was measured and cells $\left(3 \times 10^{5}\right)$ were lysed for total RNA isolation and qRT-PCR analysis of Nrf2 and GABARAPL1 mRNA levels or western blot analysis of eIF4E3 and Nrf2 protein levels as indicated. The data were normalized to ACTB mRNA levels. 


\section{Cytotoxicity assay}

Cells were treated with carfilzomib and agents at the indicated concentrations and cell growth was measured using the alamarBlue cell viability and proliferation reagent (Thermo Fisher Scientific) as previously described $[10,11]$.

\section{Measurement of NADPH}

NADPH was measured using an NADP/NADPH Quantitation Kit (Sigma-Aldrich, Cat. No. MAK038) according to the manufacturer's instructions [102].

\section{Measurement of FAO}

Oxygen consumption rate was measured using the XF24 Extracellular Flux Analyzer (Seahorse Bioscience) as previously described [103]. FAO was determined by XF Cell Mito Stress Test (Cat. No. 103010-100) using the XF Palmitate-BSA FAO substrate (Cat. No. 102720-100) in the absence or presence of etomoxir according to the manufacturer's instructions.

\section{ROS and autophagy detection}

Cells $\left(4 \times 10^{5}\right.$ per $\left.\mathrm{ml}\right)$ were seeded into 12 well plates in $500 \mu \mathrm{l}$ aliquots and treated with 0,25 or $50 \mathrm{nM}$ carfilzomib for 18 hours. $\mathrm{H}_{2} \mathrm{O}_{2}$-associated ROS levels were quantified by flow cytometry using $488 \mathrm{~nm}$ excitation and 530/30 $\mathrm{nm}$ band pass filter detection after labeling with CM- $\mathrm{H}_{2}$ DCFDA (Thermo Fisher Scientific, Cat. No. C6827) for 60 minutes at $37^{\circ} \mathrm{C} . \mathrm{O}_{2} \cdot-$ levels were quantified by flow cytometry using $488 \mathrm{~nm}$ excitation and 585/42 $\mathrm{nm}$ band pass detection after labeling with MitoSOX Red (Thermo Fisher Scientific, Cat. No. M36008) for 30 minutes at $37^{\circ} \mathrm{C}$. Flow cytometry was performed on a FACSAria instrument equipped with FACSDiva software (BD Biosciences). Dead cells were identified by SYTOX Blue staining (Thermo Fisher Scientific, Cat. No. S34857) and excluded from analysis. Data were analyzed with FlowJo Mac v10.0.2 (Tree Star) and presented on a bivariate plot versus $633 \mathrm{~nm}$ excitation and 780/60 nm band pass filter detection.

Autophagy was measured with the CytoID autophagy detection kit (Enzo, Cat. No. ENZ51031-K200) using a FACSAria instrument, and data were analyzed with FlowJo Mac v10.0.2 as previously described [11].

\section{Cellular fractionation}

Nuclear and cytoplasmic extracts were prepared for western blot analysis essentially as described [64].

\section{Confocal microscopy}

Immunofluorescence confocal microscopy was performed as previously described [11]. In brief, cells $\left(2.5 \times 10^{5}\right)$ were centrifuged onto a microscope slide at $1,000 \mathrm{rpm}$ for 5 minutes using a Shandon Cytospin 4 instrument. The cells were then immediately fixed in $3.7 \%$ formaldehyde for 5 minutes at room temperature and permeabilized with $0.5 \%$ Triton X-100 in phosphatebuffered saline (PBS) for 15 minutes at room temperature. Following permeabilization, the cells were rinsed with PBS and blocked in PBS containing 10\% goat serum and $0.01 \%$ Triton X-100 for 1 hour at room temperature. The cells were then incubated with anti-Nrf2 antibody diluted to a final concentration of $0.4 \mu \mathrm{g} / \mathrm{ml}$, in PBS containing $1 \%$ goat and $0.01 \%$ Triton $\mathrm{X}-100$ for 1 hour at room temperature. The cells were rinsed with PBS and then incubated with Alexa Fluor 488-conjugated goat antirabbit secondary antibody (Thermo Fisher Scientific, Cat. No. R37116) diluted 1:500 in PBS containing 1\% goat serum and $0.01 \%$ Triton $\mathrm{X}-100$ for 1 hour at room temperature. The cells were rinsed with PBS and mounted with Fluoromount G (Electron Microscopy Sciences). Imaging analysis was performed on a Cell Observer SD spinning disk confocal system equipped with Zen software (Carl Zeiss Microscopy).

\section{Proteasome activity}

Proteasome chymotrypsin-like activity was measured by cleavage of a specific luminogenic proteasome substrate (succinyl-leucine-leucine-valinetyrosine-aminoluciferin) using the Proteasome-Glo Chymotrypsin-Like Assay according to the manufacturer's instructions (Promega Corporation, Cat. No. G8660). Luminescence intensities were quantified with a Gemini XPS microplate spectrofluorometer equipped with SoftMax Pro software (Molecular Devices Corp.).

\section{ACKNOWLEDGMENTS}

We thank Leif Bergsagel for providing the LP-1 and KMS-11 cell lines. This work was supported by a Grant from the Dr. Cyrus and Myrtle Katzen Cancer Research Center at The George Washington University, a King Fahd Endowment from The George Washington University School of Medicine and Health Sciences, and a generous donation from Marc Cohen. 


\section{CONFLICTS OF INTEREST}

The authors declare no conflict of interest.

\section{REFERENCES}

1. Rajkumar SV, Kumar S. Multiple myeloma: diagnosis and treatment. Mayo Clin. Proc. 2016; 91: 101-119.

2. San Miguel JF, Schlag R, Khuageva NK, Dimopoulos MA, Shpilberg O, Kropff M, Spicka I, Petrucci MT, Palumbo A, Samoilova OS, Dmoszynska A, Abdulkadyrov KM, Schots R, et al. Bortezomib plus melphalan and prednisone for initial treatment of multiple myeloma. N. Engl. J. Med. 2008; 359: 906-917.

3. Richardson PG, Weller E, Lonial S, Jakubowiak AJ, Jagannath S, Raje NS, Avigan DE, Xie W, Ghobrial IM, Schlossman RL, Mazumder A, Munshi NC, Vesole DH, et al. Lenalidomide, bortezomib, and dexamethasone combination therapy in patients with newly diagnosed multiple myeloma. Blood. 2010; 116: 679-686.

4. Dolloff NG. Emerging therapeutic strategies for overcoming proteasome inhibitor resistance. Adv. Cancer Res. 2015; 127: 191-226.

5. Abdi J, Chen G, Chang H. Drug resistance in multiple myeloma: latest findings and new concepts on molecular mechanisms. Oncotarget. 2013; 4: 2186-2207. doi: 10.18632/oncotarget.1497.

6. Kim KB, Crews CM. From epoxomicin to carfilzomib: chemistry, biology, and medical outcomes. Nat. Prod. Rep. 2013; 30: 600-604

7. Herndon TM, Deisseroth A, Kaminskas E, Kane RC, Koti KM, Rothmann MD, Habtemariam B, Bullock J, Bray JD, Hawes J, Palmby TR, Jee J, Adams W, et al. U.S. Food and Drug Administration approval: carfilzomib for the treatment of multiple myeloma. Clin. Cancer Res. 2013; 19: 45594563.

8. Siegel DS, Martin T, Wang M, Vij R, Jakubowiak AJ, Lonial S, Trudel S, Kukreti V, Bahlis N, Alsina M, Chanan-Khan A, Buadi F, Reu FJ, et al. A phase 2 study of single-agent carfilzomib (PX-171-003-A1) in patients with relapsed and refractory multiple myeloma. Blood. 2012; 120: 2817-2825.

9. Hajek R, Bryce R, Ro S, Klencke B, Ludwig H. Design and rationale of FOCUS (PX-171-011): a randomized, openlabel, phase 3 study of carfilzomib versus best supportive care regimen in patients with relapsed and refractory multiple myeloma (R/R MM). BMC Cancer. 2012; 12: 4152407-12-415.

10. Hawley TS, Riz I, Yang W, Wakabayashi Y, Depalma L, Chang YT, Peng W, Zhu J, Hawley RG. Identification of an ABCB1 (P-glycoprotein)-positive carfilzomib-resistant myeloma subpopulation by the pluripotent stem cell fluorescent dye CDy1. Am. J. Hematol. 2013; 88: 265-272.

11. Riz I, Hawley TS, Hawley RG. KLF4-SQSTM1/p62- associated prosurvival autophagy contributes to carfilzomib resistance in multiple myeloma models. Oncotarget. 2015; 6: 14814-14831. doi: 10.18632/oncotarget.4530.

12. Katsuragi Y, Ichimura Y, Komatsu M. p62/SQSTM1 functions as a signaling hub and an autophagy adaptor. FEBS J. 2015; 282: 4672-4678.

13. Komatsu M, Kurokawa H, Waguri S, Taguchi K, Kobayashi A, Ichimura Y, Sou YS, Ueno I, Sakamoto A, Tong KI, Kim M, Nishito Y, Iemura S, et al. The selective autophagy substrate p62 activates the stress responsive transcription factor Nrf2 through inactivation of Keap1. Nat. Cell Biol. 2010; 12: 213-223.

14. Jain A, Lamark T, Sjottem E, Larsen KB, Awuh JA, Overvatn A, McMahon M, Hayes JD, Johansen T. p62/ SQSTM1 is a target gene for transcription factor NRF2 and creates a positive feedback loop by inducing antioxidant response element-driven gene transcription. J. Biol. Chem. 2010; 285: 22576-22591.

15. Hayes JD, Dinkova-Kostova AT. The Nrf2 regulatory network provides an interface between redox and intermediary metabolism. Trends Biochem. Sci. 2014; 39: 199-218.

16. Menegon S, Columbano A, Giordano S. The dual roles of NRF2 in cancer. Trends Mol. Med. 2016; 22: 578-593.

17. Huang Y, Li W, Su ZY, Kong AN. The complexity of the Nrf2 pathway: beyond the antioxidant response. J. Nutr. Biochem. 2015; 26: 1401-1413.

18. Kobayashi A, Kang MI, Okawa H, Ohtsuji M, Zenke Y, Chiba T, Igarashi K, Yamamoto M. Oxidative stress sensor Keap1 functions as an adaptor for Cul3-based E3 ligase to regulate proteasomal degradation of Nrf2. Mol. Cell. Biol. 2004; 24: 7130-7139.

19. Kobayashi A, Kang MI, Watai Y, Tong KI, Shibata T, Uchida K, Yamamoto M. Oxidative and electrophilic stresses activate Nrf2 through inhibition of ubiquitination activity of Keap1. Mol. Cell. Biol. 2006; 26: 221-229.

20. Baird L, Lleres D, Swift S, Dinkova-Kostova AT. Regulatory flexibility in the Nrf2-mediated stress response is conferred by conformational cycling of the Keap1-Nrf2 protein complex. Proc. Natl. Acad. Sci. U S A. 2013; 110: 15259-15264.

21. Li W, Thakor N, Xu EY, Huang Y, Chen C, Yu R, Holcik M, Kong AN. An internal ribosomal entry site mediates redox-sensitive translation of Nrf2. Nucleic Acids Res. 2010; 38: 778-788.

22. Purdom-Dickinson SE, Sheveleva EV, Sun H, Chen QM. Translational control of Nrf2 protein in activation of antioxidant response by oxidants. Mol. Pharmacol. 2007; 72: 1074-1081.

23. Zhang J, Dinh TN, Kappeler K, Tsaprailis G, Chen QM. La autoantigen mediates oxidant induced de novo Nrf2 protein translation. Mol. Cell. Proteomics. 2012; 11: M111.015032.

24. Shay KP, Michels AJ, Li W, Kong AN, Hagen TM. Cap-independent Nrf2 translation is part of a lipoic acid- 
stimulated detoxification stress response. Biochim. Biophys. Acta. 2012; 1823: 1102-1109.

25. Perez-Leal O, Barrero CA, Merali S. Translational control of Nrf2 within the open reading frame. Biochem. Biophys. Res. Commun. 2013; 437: 134-139.

26. Cullinan SB, Zhang D, Hannink M, Arvisais E, Kaufman RJ, Diehl JA. Nrf2 is a direct PERK substrate and effector of PERK-dependent cell survival. Mol. Cell. Biol. 2003; 23: 7198-7209.

27. Cullinan SB, Diehl JA. PERK-dependent activation of Nrf2 contributes to redox homeostasis and cell survival following endoplasmic reticulum stress. J. Biol. Chem. 2004; 279: 20108-20117.

28. Del Vecchio CA, Feng Y, Sokol ES, Tillman EJ, Sanduja S, Reinhardt F, Gupta PB. De-differentiation confers multidrug resistance via noncanonical PERK-Nrf2 signaling. PLoS Biol. 2014; 12: e1001945.

29. Wanders D, Stone KP, Forney LA, Cortez CC, Dille KN, Simon J, Xu M, Hotard EC, Nikonorova IA, Pettit AP, Anthony TG, Gettys TW. Role of GCN2-independent signaling through a noncanonical PERK/NRF2 pathway in the physiological responses to dietary methionine restriction. Diabetes. 2016; 65: 1499-1510.

30. Maas NL, Diehl JA. Molecular pathways: the PERKs and pitfalls of targeting the unfolded protein response in cancer. Clin. Cancer Res. 2015; 21: 675-679.

31. Harding HP, Zhang Y, Ron D. Protein translation and folding are coupled by an endoplasmic-reticulum-resident kinase. Nature. 1999; 397: 271-274.

32. Harding HP, Zhang Y, Bertolotti A, Zeng H, Ron D. Perk is essential for translational regulation and cell survival during the unfolded protein response. Mol. Cell. 2000; 5: 897-904.

33. Lu PD, Harding HP, Ron D. Translation reinitiation at alternative open reading frames regulates gene expression in an integrated stress response. J. Cell Biol. 2004; 167: 27 33.

34. Ye P, Mimura J, Okada T, Sato H, Liu T, Maruyama A, Ohyama C, Itoh K. Nrf2- and ATF4-dependent upregulation of xCT modulates the sensitivity of T24 bladder carcinoma cells to proteasome inhibition. Mol. Cell. Biol. 2014; 34: 3421-3434.

35. He CH, Gong P, Hu B, Stewart D, Choi ME, Choi AM, Alam J. Identification of activating transcription factor 4 (ATF4) as an Nrf2-interacting protein. Implication for heme oxygenase-1 gene regulation. J. Biol. Chem. 2001; 276: 20858-20865.

36. Zong ZH, Du ZX, Li N, Li C, Zhang Q, Liu BQ, Guan Y, Wang HQ. Implication of Nrf2 and ATF4 in differential induction of $\mathrm{CHOP}$ by proteasome inhibition in thyroid cancer cells. Biochim. Biophys. Acta. 2012; 1823: 13951404.

37. Osborne MJ, Volpon L, Kornblatt JA, Culjkovic-Kraljacic B, Baguet A, Borden KL. eIF4E3 acts as a tumor suppressor by utilizing an atypical mode of methyl-7-guanosine cap recognition. Proc. Natl. Acad. Sci. U S A. 2013; 110: 3877 3882 .

38. Landon AL, Muniandy PA, Shetty AC, Lehrmann E, Volpon L, Houng S, Zhang Y, Dai B, Peroutka R, MazanMamczarz K, Steinhardt J, Mahurkar A, Becker KG, et al. MNKs act as a regulatory switch for eIF4E1 and eIF4E3 driven mRNA translation in DLBCL. Nat. Commun. 2014; 5: 5413.

39. Pegoraro L, Malavasi F, Bellone G, Massaia M, Boccadoro M, Saglio G, Guerrasio A, Benetton G, Lombardi L, Coda $\mathrm{R}$. The human myeloma cell line LP-1: a versatile model in which to study early plasma-cell differentiation and c-myc activation. Blood. 1989; 73: 1020-1027.

40. Chorley BN, Campbell MR, Wang X, Karaca M, Sambandan D, Bangura F, Xue P, Pi J, Kleeberger SR, Bell DA. Identification of novel NRF2-regulated genes by ChIPSeq: influence on retinoid X receptor alpha. Nucleic Acids Res. 2012; 40: 7416-7429.

41. Malhotra D, Portales-Casamar E, Singh A, Srivastava S, Arenillas D, Happel C, Shyr C, Wakabayashi N, Kensler TW, Wasserman WW, Biswal S. Global mapping of binding sites for Nrf2 identifies novel targets in cell survival response through ChIP-Seq profiling and network analysis. Nucleic Acids Res. 2010; 38: 5718-5734.

42. Campbell MR, Karaca M, Adamski KN, Chorley BN, Wang $X$, Bell DA. Novel hematopoietic target genes in the NRF2mediated transcriptional pathway. Oxid. Med. Cell. Longev. 2013; 2013: 120305.

43. Subramanian A, Tamayo P, Mootha VK, Mukherjee S, Ebert BL, Gillette MA, Paulovich A, Pomeroy SL, Golub TR, Lander ES, Mesirov JP. Gene set enrichment analysis: a knowledge-based approach for interpreting genome-wide expression profiles. Proc. Natl. Acad. Sci. U S A. 2005; 102: 15545-15550.

44. Kwon AT, Arenillas DJ, Worsley Hunt R, Wasserman WW. oPOSSUM-3: advanced analysis of regulatory motif over-representation across genes or ChIP-Seq datasets. G3 (Bethesda). 2012; 2: 987-1002.

45. Kang HJ, Yi YW, Hong YB, Kim HJ, Jang YJ, Seong YS, Bae I. HER2 confers drug resistance of human breast cancer cells through activation of NRF2 by direct interaction. Sci. Rep. 2014; 4: 7201.

46. Khalil HS, Langdon SP, Kankia IH, Bown J, Deeni YY. NRF2 regulates HER2 and HER3 signaling pathway to modulate sensitivity to targeted immunotherapies. Oxid. Med. Cell. Longev. 2016; 2016: 4148791.

47. Han J, Back SH, Hur J, Lin YH, Gildersleeve R, Shan J, Yuan CL, Krokowski D, Wang S, Hatzoglou M, Kilberg MS, Sartor MA, Kaufman RJ. ER-stress-induced transcriptional regulation increases protein synthesis leading to cell death. Nat. Cell Biol. 2013; 15: 481-490.

48. Li Z, Qi CF, Shin DM, Zingone A, Newbery HJ, Kovalchuk AL, Abbott CM, Morse HC,3rd. Eef1a2 promotes cell growth, inhibits apoptosis and activates JAK/STAT and 
AKT signaling in mouse plasmacytomas. PLoS One. 2010; 5: e10755.

49. Villalonga P, Fernandez de Mattos S, Ridley AJ. RhoE inhibits 4E-BP1 phosphorylation and eIF4E function impairing cap-dependent translation. J. Biol. Chem. 2009; 284: 35287-35296.

50. Sun GD, Kobayashi T, Abe M, Tada N, Adachi H, Shiota A, Totsuka Y, Hino O. The endoplasmic reticulum stressinducible protein Niban regulates eIF2 $\alpha$ and S6K1/4E-BP1 phosphorylation. Biochem. Biophys. Res. Commun. 2007; 360: 181-187.

51. Le Grand JN, Chakrama FZ, Seguin-Py S, Fraichard A, Delage-Mourroux R, Jouvenot M, Boyer-Guittaut M. GABARAPL1 (GEC1): original or copycat? Autophagy. 2011; 7: 1098-1107.

52. Broos S, Hulpiau P, Galle J, Hooghe B, Van Roy F, De Bleser P. ConTra v2: a tool to identify transcription factor binding sites across species, update 2011. Nucleic Acids Res. 2011; 39: W74-8.

53. Behrends C, Sowa ME, Gygi SP, Harper JW. Network organization of the human autophagy system. Nature. 2010; 466: 68-76.

54. Weidberg H, Shvets E, Shpilka T, Shimron F, Shinder V, Elazar Z. LC3 and GATE-16/GABARAP subfamilies are both essential yet act differently in autophagosome biogenesis. EMBO J. 2010; 29: 1792-1802.

55. Klionsky DJ, Abdelmohsen $\mathrm{K}$, Abe A, Abedin MJ, Abeliovich H, Acevedo Arozena A, Adachi H, Adams CM, Adams PD, Adeli K, Adhihetty PJ, Adler SG, Agam G, et al. Guidelines for the use and interpretation of assays for monitoring autophagy (3rd edition). Autophagy. 2016; 12: $1-222$.

56. Wu KC, Cui JY, Klaassen CD. Beneficial role of Nrf2 in regulating NADPH generation and consumption. Toxicol. Sci. 2011; 123: 590-600.

57. Ludtmann MH, Angelova PR, Zhang Y, Abramov AY, Dinkova-Kostova AT. Nrf2 affects the efficiency of mitochondrial fatty acid oxidation. Biochem. J. 2014; 457: 415-424.

58. Singh R, Kaushik S, Wang Y, Xiang Y, Novak I, Komatsu M, Tanaka K, Cuervo AM, Czaja MJ. Autophagy regulates lipid metabolism. Nature. 2009; 458: 1131-1135.

59. Lee EA, Angka L, Rota SG, Hanlon T, Mitchell A, Hurren R, Wang XM, Gronda M, Boyaci E, Bojko B, Minden M, Sriskanthadevan S, Datti A, et al. Targeting mitochondria with avocatin B induces selective leukemia cell death. Cancer Res. 2015; 75: 2478-2488.

60. Kirkland RA, Saavedra GM, Franklin JL. Rapid activation of antioxidant defenses by nerve growth factor suppresses reactive oxygen species during neuronal apoptosis: evidence for a role in cytochrome c redistribution. J. Neurosci. 2007; 27: 11315-11326.

61. Pike LS, Smift AL, Croteau NJ, Ferrick DA, Wu M. Inhibition of fatty acid oxidation by etomoxir impairs
NADPH production and increases reactive oxygen species resulting in ATP depletion and cell death in human glioblastoma cells. Biochim. Biophys. Acta. 2011; 1807 : 726-734.

62. Murata H, Takamatsu H, Liu S, Kataoka K, Huh NH, Sakaguchi M. NRF2 regulates PINK1 expression under oxidative stress conditions. PLoS One. 2015; 10: e0142438.

63. Arlt A, Sebens S, Krebs S, Geismann C, Grossmann M, Kruse ML, Schreiber S, Schafer H. Inhibition of the Nrf2 transcription factor by the alkaloid trigonelline renders pancreatic cancer cells more susceptible to apoptosis through decreased proteasomal gene expression and proteasome activity. Oncogene. 2013; 32: 4825-4835.

64. Kang KW, Lee SJ, Park JW, Kim SG. Phosphatidylinositol 3-kinase regulates nuclear translocation of NF-E2-related factor 2 through actin rearrangement in response to oxidative stress. Mol. Pharmacol. 2002; 62: 1001-1010.

65. Acosta-Alvear D, Cho MY, Wild T, Buchholz TJ, Lerner AG, Simakova O, Hahn J, Korde N, Landgren O, Maric I, Choudhary C, Walter P, Weissman JS, et al. Paradoxical resistance of multiple myeloma to proteasome inhibitors by decreased levels of 19S proteasomal subunits. Elife. 2015; 4: e08153.

66. Avivar-Valderas A, Bobrovnikova-Marjon E, Alan Diehl J, Bardeesy N, Debnath J, Aguirre-Ghiso JA. Regulation of autophagy during ECM detachment is linked to a selective inhibition of mTORC1 by PERK. Oncogene. 2013; 32: 4932-4940.

67. Axten JM, Romeril SP, Shu A, Ralph J, Medina JR, Feng Y, Li WH, Grant SW, Heerding DA, Minthorn E, Mencken T, Gaul N, Goetz A, et al. Discovery of GSK2656157: an optimized PERK inhibitor selected for preclinical development. ACS Med. Chem. Lett. 2013; 4: 964-968.

68. Sidrauski C, Acosta-Alvear D, Khoutorsky A, Vedantham P, Hearn BR, Li H, Gamache K, Gallagher CM, Ang KK, Wilson C, Okreglak V, Ashkenazi A, Hann B, et al. Pharmacological brake-release of mRNA translation enhances cognitive memory. Elife. 2013; 2: e00498.

69. Wethmar K, Schulz J, Muro EM, Talyan S, AndradeNavarro MA, Leutz A. Comprehensive translational control of tyrosine kinase expression by upstream open reading frames. Oncogene. 2016; 35: 1736-1742.

70. Feng YX, Sokol ES, Del Vecchio CA, Sanduja S, Claessen JH, Proia TA, Jin DX, Reinhardt F, Ploegh HL, Wang Q, Gupta PB. Epithelial-to-mesenchymal transition activates PERK-eIF2alpha and sensitizes cells to endoplasmic reticulum stress. Cancer. Discov. 2014; 4: 702-715.

71. Kim WD, Kim YW, Cho IJ, Lee CH, Kim SG. E-cadherin inhibits nuclear accumulation of Nrf2: implications for chemoresistance of cancer cells. J. Cell. Sci. 2012; 125: 1284-1295.

72. Arfmann-Knubel S, Struck B, Genrich G, Helm O, Sipos B, Sebens S, Schafer H. The crosstalk between Nrf2 and TGF- $\beta 1$ in the epithelial-mesenchymal transition 
of pancreatic duct epithelial cells. PLoS One. 2015; 10: e0132978.

73. Sekiyama N, Arthanari H, Papadopoulos E, Rodriguez-Mias RA, Wagner G, Leger-Abraham M. Molecular mechanism of the dual activity of 4EGI-1: dissociating eIF4G from eIF4E but stabilizing the binding of unphosphorylated 4E-BP1. Proc. Natl. Acad. Sci. U S A. 2015; 112: E4036-E4045.

74. Joshi B, Cameron A, Jagus R. Characterization of mammalian eIF4E-family members. Eur. J. Biochem. 2004; 271: 2189-2203.

75. Siddiqui N, Sonenberg N. Signalling to eIF4E in cancer. Biochem. Soc. Trans. 2015; 43: 763-772.

76. Paiva B, Corchete LA, Vidriales MB, Puig N, Maiso P, Rodriguez I, Alignani D, Burgos L, Sanchez ML, Barcena P, Echeveste MA, Hernandez MT, Garcia-Sanz R, et al. Phenotypic and genomic analysis of multiple myeloma minimal residual disease tumor cells: a new model to understand chemoresistance. Blood. 2016; 127: 1896-1906.

77. Keats JJ, Chesi M, Egan JB, Garbitt VM, Palmer SE, Braggio E, Van Wier S, Blackburn PR, Baker AS, Dispenzieri A, Kumar S, Rajkumar SV, Carpten JD, et al. Clonal competition with alternating dominance in multiple myeloma. Blood. 2012; 120: 1067-1076.

78. Chesi M, Nardini E, Lim RS, Smith KD, Kuehl WM, Bergsagel PL. The $\mathrm{t}(4 ; 14)$ translocation in myeloma dysregulates both FGFR3 and a novel gene, MMSET, resulting in IgH/MMSET hybrid transcripts. Blood. 1998; 92: 3025-3034.

79. Agnelli L, Forcato M, Ferrari F, Tuana G, Todoerti K, Walker BA, Morgan GJ, Lombardi L, Bicciato S, Neri A. The reconstruction of transcriptional networks reveals critical genes with implications for clinical outcome of multiple myeloma. Clin. Cancer Res. 2011; 17: 7402-7412.

80. Shaughnessy JD,Jr, Zhan F, Burington BE, Huang Y, Colla S, Hanamura I, Stewart JP, Kordsmeier B, Randolph C, Williams DR, Xiao Y, Xu H, Epstein J, et al. A validated gene expression model of high-risk multiple myeloma is defined by deregulated expression of genes mapping to chromosome 1. Blood. 2007; 109: 2276-2284.

81. Goswami CP, Nakshatri H. PROGgeneV2: enhancements on the existing database. BMC Cancer. 2014; 14: 970-240714-970.

82. Azab AK, Hu J, Quang P, Azab F, Pitsillides C, Awwad R, Thompson B, Maiso P, Sun JD, Hart CP, Roccaro AM, Sacco A, Ngo HT, et al. Hypoxia promotes dissemination of multiple myeloma through acquisition of epithelial to mesenchymal transition-like features. Blood. 2012; 119: 5782-5794.

83. Takamiya R, Takahashi M, Uehara Y, Ariki S, Hashimoto J, Hasegawa Y, Kuroki Y. The single N-glycan deletion mutant of soluble ErbB3 protein attenuates heregulin $\beta 1$ induced tumor progression by blocking of the HIF-1 and Nrf2 pathway. Biochem. Biophys. Res. Commun. 2014;
454: 364-368.

84. Grigore AD, Jolly MK, Jia D, Farach-Carson MC, Levine H. Tumor Budding: The name is EMT. Partial EMT. J. Clin. Med. 2016; 5: E51.

85. Zhang XD, Baladandayuthapani V, Lin H, Mulligan G, Li B, Esseltine DL, Qi L, Xu J, Hunziker W, Barlogie B, Usmani SZ, Zhang Q, Crowley J, et al. Tight junction protein 1 modulates proteasome capacity and proteasome inhibitor sensitivity in multiple myeloma via EGFR/JAK1/ STAT3 signaling. Cancer Cell. 2016; 29: 639-652.

86. Zeisberg M, Neilson EG. Biomarkers for epithelialmesenchymal transitions. J. Clin. Invest. 2009; 119: 14291437.

87. Zhao J, Moore AN, Redell JB, Dash PK. Enhancing expression of Nrf2-driven genes protects the blood brain barrier after brain injury. J. Neurosci. 2007; 27: 1024010248.

88. Shaykhiev R, Zuo WL, Chao I, Fukui T, Witover B, Brekman A, Crystal RG. EGF shifts human airway basal cell fate toward a smoking-associated airway epithelial phenotype. Proc. Natl. Acad. Sci. U S A. 2013; 110: 1210212107.

89. Chen X, Yeung TK, Wang Z. Enhanced drug resistance in cells coexpressing ErbB2 with EGF receptor or ErbB3. Biochem. Biophys. Res. Commun. 2000; 277: 757-763.

90. Yaccoby S. The phenotypic plasticity of myeloma plasma cells as expressed by dedifferentiation into an immature, resilient, and apoptosis-resistant phenotype. Clin. Cancer Res. 2005; 11: 7599-7606.

91. Chaidos A, Barnes CP, Cowan G, May PC, Melo V, Hatjiharissi E, Papaioannou M, Harrington H, Doolittle H, Terpos E, Dimopoulos M, Abdalla S, Yarranton H, et al. Clinical drug resistance linked to interconvertible phenotypic and functional states of tumor-propagating cells in multiple myeloma. Blood. 2013; 121: 318-328.

92. Leung-Hagesteijn C, Erdmann N, Cheung G, Keats JJ, Stewart AK, Reece DE, Chung KC, Tiedemann RE. Xbp1s-negative tumor B cells and pre-plasmablasts mediate therapeutic proteasome inhibitor resistance in multiple myeloma. Cancer Cell. 2013; 24: 289-304.

93. Perez-Galan P, Mora-Jensen H, Weniger MA, Shaffer AL,3rd, Rizzatti EG, Chapman CM, Mo CC, Stennett LS, Rader C, Liu P, Raghavachari N, Stetler-Stevenson $\mathrm{M}$, Yuan C, et al. Bortezomib resistance in mantle cell lymphoma is associated with plasmacytic differentiation. Blood. 2011; 117: 542-552.

94. Stessman HA, Baughn LB, Sarver A, Xia T, Deshpande R, Mansoor A, Walsh SA, Sunderland JJ, Dolloff NG, Linden MA, Zhan F, Janz S, Myers CL, et al. Profiling bortezomib resistance identifies secondary therapies in a mouse myeloma model. Mol. Cancer Ther. 2013; 12: 11401150 .

95. Li B, Fu J, Chen P, Ge X, Li Y, Kuiatse I, Wang H, Wang $\mathrm{H}$, Zhang X, Orlowski RZ. The nuclear factor (erythroid- 
derived 2)-like 2 and proteasome maturation protein axis mediate bortezomib resistance in multiple myeloma. J. Biol. Chem. 2015; 290: 29854-29868.

96. Chen S, Blank JL, Peters T, Liu XJ, Rappoli DM, Pickard MD, Menon S, Yu J, Driscoll DL, Lingaraj T, Burkhardt AL, Chen W, Garcia K, et al. Genome-wide siRNA screen for modulators of cell death induced by proteasome inhibitor bortezomib. Cancer Res. 2010; 70: 4318-4326.

97. Mancino M, Grosso S, Terragna C, Borsi E, Cavo M, Biffo S. Cap dependent translation contributes to resistance of myeloma cells to bortezomib. Translation (Austin). 2013; 1: e27245.

98. Zismanov V, Attar-Schneider O, Lishner M, Heffez Aizenfeld R, Tartakover Matalon S, Drucker L. Multiple myeloma proteostasis can be targeted via translation initiation factor eIF4E. Int. J. Oncol. 2015; 46: 860-870.

99. Soriano GP, Besse L, Li N, Kraus M, Besse A, Meeuwenoord N, Bader J, Everts B, den Dulk H, Overkleeft HS, Florea BI, Driessen C. Proteasome inhibitor-adapted myeloma cells are largely independent from proteasome activity and show complex proteomic changes, in particular in redox and energy metabolism. Leukemia. 2016; doi: 10.1038/leu.2016.102.
100. Hawley RG. The cancer stem cell conundrum in multiple myeloma. J. Stem Cell Res. Ther. 2012; 2: 1000e110.

101. Keats JJ, Fonseca R, Chesi M, Schop R, Baker A, Chng WJ, Van Wier S, Tiedemann R, Shi CX, Sebag M, Braggio E, Henry T, Zhu YX, et al. Promiscuous mutations activate the noncanonical NF- $\kappa$ B pathway in multiple myeloma. Cancer Cell. 2007; 12: 131-144.

102. Desideri E, Vegliante R, Cardaci S, Nepravishta R, Paci M, Ciriolo MR. MAPK14/p38 $\alpha$-dependent modulation of glucose metabolism affects ROS levels and autophagy during starvation. Autophagy. 2014; 10: 1652-1665.

103. Dalva-Aydemir S, Bajpai R, Martinez M, Adekola KU, Kandela I, Wei C, Singhal S, Koblinski JE, Raje NS, Rosen ST, Shanmugam M. Targeting the metabolic plasticity of multiple myeloma with FDA-approved ritonavir and metformin. Clin. Cancer Res. 2015; 21: 1161-1171. 